\title{
Global auroral conductance distribution due to electron and proton precipitation from IMAGE-FUV observations
}

\author{
V. Coumans ${ }^{1}$, J.-C. Gérard ${ }^{1}$, B. Hubert ${ }^{1}$, M. Meurant ${ }^{1}$, and S. B. Mende ${ }^{2}$ \\ ${ }^{1}$ Laboratoire de Physique Atmosphérique et Planétaire, Université de Liège, B5C, Allée du 6 Août 17, 4000 Liège, Belgium \\ ${ }^{2}$ SSL, University of California, Berkeley, Centennial Drive at Grizzly Peak Blvd, CA 94720-7450, USA
}

Received: 12 June 2003 - Revised: 21 November 2003 - Accepted: 11 December 2003 - Published: 8 April 2004

\begin{abstract}
The Far Ultraviolet (FUV) imaging system on board the IMAGE satellite provides a global view of the north auroral region in three spectral channels, including the SI12 camera sensitive to Doppler shifted Lyman- $\alpha$ emission. FUV images are used to produce instantaneous maps of electron mean energy and energy fluxes for precipitated protons and electrons. We describe a method to calculate ionospheric Hall and Pedersen conductivities induced by auroral proton and electron ionization based on a model of interaction of auroral particles with the atmosphere. Different assumptions on the energy spectral distribution for electrons and protons are compared. Global maps of ionospheric conductances due to instantaneous observation of precipitating protons are calculated. The contribution of auroral protons in the total conductance induced by both types of auroral particles is also evaluated and the importance of proton precipitation is evaluated. This method is well adapted to analyze the time evolution of ionospheric conductances due to precipitating particles over the auroral region or in particular sectors. Results are illustrated with conductance maps of the north polar region obtained during four periods with different activity levels. It is found that the proton contribution to conductance is relatively higher during quiet periods than during substorms. The proton contribution is higher in the period before the onset and strongly decreases during the expansion phase of substorms. During a substorm which occurred on 28 April 2001, a region of strong proton precipitation is observed with SI12 around 14:00 MLT at $\sim 75^{\circ}$ MLAT. Calculation of conductances in this sector shows that neglecting the protons contribution would produce a large error. We discuss possible effects of the proton precipitation on electron precipitation in auroral arcs. The increase in the ionospheric conductivity, induced by a former proton precipitation can reduce the potential drop along field lines in the upward field-aligned currents by creating an opposite polarization electric field. This feedback mechanism possibly reduces the electron acceleration.
\end{abstract}

Key words. Ionosphere (auroral ionosphere; ionospheremagnetosphere interactions; particle precipitation)

Correspondence to: V. Coumans

(v.coumans@ulg.ac.be)

\section{Introduction}

The calculation of the ionosphere height integrated conductivities is important in evaluating the ionosphere electrodynamics and in modeling the interactions between magnetosphere, ionosphere and thermosphere. In particular, the Pedersen conductance is useful to calculate the Joule heating rate. Thus, it is crucial in studies of the ionospheric electrodynamics to be able to determine the global distribution of conductance and its time evolution.

Conductivities are usually estimated from the electron density height profile which is controlled by the EUV solar radiation and the auroral precipitation. One experimental approach is to use the height profile of electron density measured from the ground with incoherent-scatter radars, such as the Chatanika or EISCAT radars, or derived from scanning photometers data. Horwitz et al. (1978) and Vickrey et al. $(1981,1982)$ provided examples of ionospheric Hall and Pedersen conductances evaluated from Chatanika radar measurements. Mende et al. (1984) compared the conductances derived from auroral spectroscopic measurements obtained with meridian scanning photometers and those derived from the incoherent scatter radar at Chatanika. Robinson et al. (1989) compared the latter with the auroral luminosities observed with the auroral imagers on board the DEI satellite. EISCAT radar measurements of the electron density were used by Schlegel (1988), Brekke et al. $(1988 ; 1989)$ and Lester et al. (1996) to calculate ionospheric conductances. Another method to evaluate ionospheric conductances from the ground uses observations of $\mathbf{B}$ and $\mathbf{E}$ fields from, for example, the STARE coherent radar system and the IMS Scandinavian Magnetometer Array (Inhester et al., 1992).

In situ measurements also allow one to calculate conductances. Evans et al. (1977) determined the Hall and Pedersen conductivities of the auroral ionosphere produced by the particle precipitation from the auroral electron data obtained during the flight of Polar 3 over an auroral arc. Vondrak and Robinson (1985) compared the height-integrated conductivities inferred from the AE-C satellite measurements and those computed from the Chatanika radar measurements. These studies were localized in space since the instruments have restricted fields-of-view which do not permit observations of 
the auroral zone at all latitudes and local times. An effort was done to extrapolate the EISCAT Pedersen conductances to other parts of the sky (Kosch et al., 1998).

In order to properly estimate the instantaneous threedimensional current system, its variations during auroral substorms and the spatial distribution of Joule heating, an accurate knowledge of instantaneous ionospheric conductances over the entire polar region is required. For electrons three approaches have been applied so far.

1) Several empirical models of conductances were developed and validated with in situ particle measurements. These empirical formulas for $\Sigma_{\mathrm{P}}$ and $\Sigma_{\mathrm{H}}$ depend on the mean energy and the energy flux of the incident particles. Spiro et al. (1982) used data from the AE-C and AE-D particle detectors to develop an empirical model of electron auroral zone conductances from patterns of energy influx and characteristic energy. The global distribution of the auroral enhancement portion of the Pedersen and Hall conductances was inferred from the data by means of empirical fits to detailed energy deposition calculations. Robinson et al. (1987) estimated the validity of their empirical conductances formulas using precipitating electron measurements made by the Hilat satellite during a pass over Greenland. Gjerloev and Hoffman (2000a, b) evaluated Hall and Pedersen conductances from 31 individual DE-2 substorm crossing measurements using a monoenergetic conductance model, which divides the energy flux into energy bands each centered at the energy $E_{i}$ and then integrates over the energy spectrum. From these results, Gjerloev and Hoffman, (2000c) developed an empirical model of conductances.

2) Statistical conductivity patterns have been constructed on the basis of statistical maps of auroral precipitation. Wallis and Budzinski (1981) presented patterns based on ISIS 2 satellite data. Hardy et al. (1987) used the Hardy et al. (1985) statistical global patterns of the integral energy flux and average energy of precipitating auroral electrons, to determine the global pattern of electron-produced, heightintegrated Hall and Pedersen conductivities. This approach cannot provide an accurate picture of the instantaneous effect of the auroral precipitation on the ionosphere since they only depend on geomagnetic activity through the 3 -hour $K_{p}$ index. Fuller-Rowell and Evans (1987) used the particle influx into the atmosphere monitored by the series of polar-orbiting National Oceanic and Atmospheric Administration TIROS, NOAA 6, and NOAA 7 spacecraft, to construct statistical global patterns of height-integrated Pedersen and Hall conductivities for a discrete set of auroral activity ranges.

3) Auroral images acquired from high altitude, such as those from the Dynamic Explorer 1 satellite, coupled with an auroral model, provide instantaneous ionospheric conductance patterns. Lummerzheim et al. (1991) constructed maps from DE-1 data and Rees et al. (1988)'s model.

In global models of auroral precipitation-induced conductances (Wallis and Budzinski, 1981; Spiro et al., 1982; Fuller-Rowell and Evans, 1987; Hardy et al., 1987) energetic protons have been either neglected or treated as if they were electrons. However, several studies (Basu et al., 1987; Ga- land et al., 1999; Coumans et al., 2002) have shown that protons play a major role on the ionospheric densities at given locations and times. Recently, Galand et al. (2001a) proposed a simple way to parameterize the Pedersen and Hall conductances due to auroral proton precipitation and applied them to the Hardy et al. (1989) statistical patterns of precipitating proton flux and mean energy (Galand et al., 2001b). The derivation is based on a proton transport code to calculate the electron production rate, which assumes a Maxwellian distribution of the incident proton energy flux. This approximation may, however, underestimate the highenergy tail of precipitating protons (Decker et al., 1996; Codrescu et al., 1997). Since Galand et al. (2001b)'s results are based on statistical patterns of precipitating particle characteristics, their conductances cannot show the time variation due to auroral particles. Those patterns are only related to geomagnetic activity through 9 levels of $K_{p}$ index.

Liou et al. (2001) performed a statistical study of the seasonal variation of auroral electron precipitation using POLAR UVI observations. Their results indicated that dayside and nightside regions of electron aurora reveal different seasonal effects which are local time and latitude dependent. First, they showed that the nightside auroral power is suppressed in summer while the dayside auroral power is enhanced and reveals the so-called post-noon auroral bright spot in the sunlit hemisphere. Second, they concluded that the energy of the precipitating electrons is higher in winter than in summer, and third, that the electron number flux is smaller in winter than in summer. Based on DMSP satellite measurements, Newell et al. (1996) also emphasized the suppression of discrete electron aurora in sunlit regions. They interpreted the observations in terms of an ionospheric conductivity feedback mechanism (Atkinson, 1970; Sato, 1978; Lysak, 1986). The ionospheric "background conductance", which is an approximately steady ionospheric conductance, which is largely due to solar ionization, plays a role in the creation of the aurora. In this study we also examine if conductances due the auroral proton precipitation can also influence the electron precipitation as the background ionospheric conductance. If the proton precipitation produces an important enhancement in the ionospheric conductances, the feedback conductivity mechanism may produce or reduce the acceleration of auroral electrons.

We propose to evaluate instantaneous conductance global distribution from IMAGE-FUV observations separately for electrons and for protons. The IMAGE (Imager for Magnetopause-to-Aurora Global Exploration) satellite, launched in March 2000, is a mission for remote sensing simultaneously of all regions of the Earth's magnetosphere. On board the IMAGE satellite, the FUV imaging system globally observes the north auroral region in the far ultraviolet (Mende et al., 2000). The imagers are designed to monitor the electron and proton precipitation and discriminate between the two types of particles. Previous studies have shown the quantitative and qualitative validity of FUV instruments from comparisons with in situ auroral particle measurements (Frey et al., 2001; Gérard et al., 2001; Coumans et al., 2002). 
Section 2 briefly describes the method used to extract the particle energy and energy flux characteristics from the FUV observations. In Sect. 3 the conductance model we developed is described step-by-step. The validation of the conductivity model and of the complete method to construct conductance maps is discussed in Sect. 4. We present some case studies in Sect. 5. We show instantaneous conductance maps and discuss the relative importance of proton-induced conductivities. In Sect. 6 we show some results obtained by using electron mean energy evaluated from FUV observations and we discuss the reliability and the relevance of this method. Finally, Sect. 7 summarizes the method and discusses the role of proton induced conductances and its possible effect on field-aligned currents associated with discrete electron aurora.

\section{The FUV imagers and the auroral precipitation}

The FUV experiment on board the IMAGE satellite includes three different imagers: the Wideband Imaging Camera (WIC) and two Spectrographic Imagers (SI12 and SI13). One characteristic of the FUV imager is its capability to simultaneously observe the Northern Hemisphere in all three spectral regions. The Spectrographic Imager is a narrowband imager of far ultraviolet auroral emissions at $121.8 \mathrm{~nm}$ and $135.6 \mathrm{~nm}$. The SI12 imager measures the brightness of the Doppler shifted Lyman- $\alpha$ auroral emission. SI12 is the first FUV imager able to detect incident proton flux without contribution from electrons. SI13 images the OI $135.6 \mathrm{~nm}$ line produced by incident primary electrons and protons and secondary electrons colliding with neutral atoms. The WIC imager has a passband between $140-180 \mathrm{~nm}$ with a low sensitivity below $140 \mathrm{~nm}$ which covers a spectral region including emissions excited by both protons and electrons. It is mostly sensitive to the LBH bands and the $149.3 \mathrm{~nm}$ NI line with small contributions from the NI $174.3 \mathrm{~nm}$ doublet and the OI $135.6 \mathrm{~nm}$ line. More information about the IMAGE mission can be found in Burch (2000) and the FUV experiment is described in Mende et al. (2000).

Frey et al. (2001) used in situ measurements from two orbits of the FAST satellite to model the auroral brightness and compared them with simultaneous FUV-WIC observations. They concluded a good agreement between predicted and observed emission rates, both in morphology and in intensity. Gérard et al. (2001) presented a comparison with SI12 observations for FAST and DMSP satellite overflights. This comparison showed an underestimation of the simulated SI12 response with the latitudinally integrated brightness. This discrepancy was tentatively attributed to highenergy protons above the $30 \mathrm{keV}$ upper limit of the detectors, suggesting that higher energy protons could be important in producing this emission. Coumans et al. (2002) used in situ particle measurements from NOAA-15 and NOAA-16 satellites to check this possibility and assess the role of proton excitation of FUV emissions. The NOAA sets of detectors offers the advantage of observing electrons in the energy range
$50 \mathrm{eV}-1000 \mathrm{keV}$ and protons from $50 \mathrm{eV}$ to $800 \mathrm{keV}$, including all relevant auroral energies. The comparison was made for both WIC and SI12 data. Taking into consideration all possible sources of error, WIC observations showed agreement with in situ induced auroral brightness within $70 \%$, but SI12 observations coupled with simulation from the Monte Carlo code predicted proton fluxes twice as large as the in situ measurements (Coumans et al., 2002). Consequently, in this work, the proton energy fluxes are adjusted by a factor of 2 to account for this overestimate of the SI12 derived proton flux.

Using proton and electron transport models we can extract the proton and electron energy flux from auroral brightness images of, respectively, the WIC and SI12 imagers. This procedure requires hypothesis on the particle mean energy. The method to extract the precipitating electron energy flux is based on simulations with the GLOW model (Solomon et al., 1988) extended to higher energies for auroral calculations (Hubert et al., 2001). This model, based on a two-stream approximation, calculates the auroral electron energy degradation and excitation by electron-induced process. The electron energy distribution is assumed to be Maxwellian. The electron energy flux patterns are calculated from WIC observations, the electron transport code and the mean energy of electron. The proton energy transport code was described in detail by Gérard et al. (2000). It is based on the direct Monte Carlo method (Marov et al., 1997), which is a stochastic implementation of the solution of the Boltzmann equations, applied to the $\mathrm{H}^{+}-\mathrm{H}$ beam.

The main limitation in this work is that the particle mean energy is not available. We consider the electron and proton mean energy patterns from Hardy et al. $(1985 ; 1989)$. These maps were computed from a statistical study using data collected over several years with detectors on board the Defense Meteorological Satellite Program (DMSP) satellites. The empirical model depends on the magnetic activity indexed by $K_{p}$. We discuss later the relevance of this approximation. For electrons, the combined WIC and SI13 simultaneous observations make it possible to evaluate the mean energy. A discussion of this method and some results are given in Sect. 6. In a first step, since SI12 only images proton precipitation, we use the SI12 data to remove the proton contribution from the WIC and SI13 images. Then from the proton transport code and with some assumptions about the proton mean energy we calculate the proton energy flux maps from SI12 images. Another difficulty stems from the airglow contribution in the WIC and SI13 observations. This contribution must be removed before deriving the precipitating flux, since we seek evaluation of the contribution of auroral particles only. This important step when using the WIC and SI13 observations to evaluate the electron mean energy is discussed in Sect. 6. The background removal was done using the method described by Immel et al. (2000). The airglow is determined from an average quiet-time airglow, individually for each instrument. 


\section{The conductance model}

From the electron and proton averaged energy and the energy flux maps, we developed a model to calculate the Hall and Pedersen conductances. The method can be separated into different steps. First, the ionospheric ionization rate is evaluated from the incident particle characteristics. We then compute the electron and ion density profiles. The last step is the calculation of the conductances.

\subsection{Ionization rates}

The calculation of the ionization height profiles requires the use of particle transport and energy degradation models described in the previous section. For electrons, the twostreams code calculates the secondary electron production rate from an incident energy flux and a mean energy of electrons, which is equivalent to the total ionization rate due to electrons. The calculations are made for a range of different mean energies and an incident energy flux of $1 \mathrm{~mW} / \mathrm{m}^{2}$. We multiply by the energy flux to evaluate the total ionization profile due to precipitating electrons.

For protons, the Monte Carlo code gives the primary electron production rate which can be introduced in the GLOW model, to estimate the secondary electron population. The sum of the primary and secondary electron production rates is the total ionization rate. The calculations are made for different energy bands, with the central energy as particle mean energy and for an energy flux of $1 \mathrm{~mW} / \mathrm{m}^{2}$. The energy distribution of incident protons is assumed to be a Kappa function with $\kappa=3.5$, as recommended by Hubert et al. (2001). The ionization profiles for each monoenergetic band are added to reconstruct the total contribution of the Kappa distribution. In this way, we obtain the total ionization profile for an energy flux of $1 \mathrm{~mW} / \mathrm{m}^{2}$ and multiply it by the actual incident energy flux, to evaluate the total ionization profile due to precipitating protons. The total ionization height profile due to the total incident charged particle is the sum of the electron and proton ionization height profiles.

\subsection{Electron and ion density profiles}

The electron density is derived from the continuity equations for the electron concentration $\mathrm{N}_{\mathrm{e}}$ :

$\frac{d N_{e}}{d t}=q_{e}-l_{e}-\operatorname{div}\left(N_{e} \boldsymbol{V}\right)$,

where $\mathrm{q}_{\mathrm{e}}$ is the electron production term, $\mathrm{l}_{\mathrm{e}}$ is the electron loss term and the third term describes the change due to transport, if the transport processes result in a net drift velocity $\mathbf{V}$. The transport term is neglected, since the diffusion time in the E- and F1-regions exceeds the time between collisions. At steady state:

$q_{e}=l_{e}=\bar{\alpha} N_{e}^{2}$

and

$$
N_{e}=\sqrt{\frac{q_{e}}{\bar{\alpha}}}
$$

where $\bar{\alpha}$ is the effective recombination coefficient. The electron density height profile is calculated from Eq. (3), using the calculated electron production profile.

The mean recombination coefficient is derived from

$\bar{\alpha}=\frac{\sum_{i} \alpha_{i}\left(T_{e}\right) N_{i}}{N_{e}}$,

where i indicates $\mathrm{N}_{2}^{+}, \mathrm{O}_{2}^{+}$or $\mathrm{NO}^{+}, \mathrm{N}_{\mathrm{i}}$ is the density of the ion species $\mathrm{i}$ and $\alpha_{\mathrm{i}}$ is the individual ion recombination coefficient. We use the ion proportion from the International Reference Atmosphere-1990 (IRI-90) model (Bilitza, 1990) to obtain the ion density $\mathrm{N}_{\mathrm{i}}$ from the electron density $\mathrm{N}_{\mathrm{e}}$. The ion recombination coefficients as a function of the electron temperature are from Rees (1989) and the electron temperature is taken from the MSIS-90 model atmosphere (Hedin et al., 1991) using the approximation that the electron temperature is equal to the neutral particle temperature.

\subsection{Conductivity profiles}

The Pedersen and Hall conductivities height profiles are computed from :

$\sigma_{P}=\frac{N_{e} e}{B}\left(\frac{v_{e n} \omega_{e}}{v_{e n}^{2}+\omega_{e}^{2}}+\frac{v_{i n} \omega_{i}}{v_{i n}^{2}+\omega_{i}^{2}}\right)$
$\sigma_{H}=\frac{N_{e} e}{B}\left(\frac{\omega_{e}^{2}}{v_{e n}^{2}+\omega_{e}^{2}}-\frac{\omega_{i}^{2}}{v_{i n}^{2}+\omega_{i}^{2}}\right)$

where conductivities are in mho $\mathrm{m}^{-1}$ or in $\mathrm{S} \mathrm{m}^{-1}$, e is the electron charge, $\mathrm{B}$ is the magnitude of the geomagnetic field, $\mathrm{N}_{\mathrm{e}}$ is the electron density, $\omega_{\mathrm{i}}$ and $\omega_{\mathrm{e}}$ the angular gyrofrequencies of, respectively, ions and electrons in the geomagnetic field. The ion gyrofrequency is calculated using an averaged mass evaluated for each altitude step. This mean ion mass is computed from the IRI-90 ion proportions and the mass of the ions. The geomagnetic field B is calculated from the International Geomagnetic Reference Field model (IGRF-2000) (Olsen, 2000) for the appropriate geographic position. $v_{\text {in }}$ and $v_{\text {en }}$ are the collision frequencies between the ion or the electron and the neutral species (Rishbeth and Garriott, 1969). $v_{\mathrm{en}}$ depends on the neutral density $\mathrm{N}_{\mathrm{n}}$ and on the temperature $\mathrm{T}$, which is assumed equal for all species. The neutral atmosphere $\mathrm{O}, \mathrm{O}_{2}$ and $\mathrm{N}_{2}$ densities are given by the MSIS-90 model atmosphere (Hedin et al., 1991).

The conductivity profiles and the conductances are computed for electrons and protons of different mean energy for an incident energy flux of $1 \mathrm{~mW} / \mathrm{m}^{2}$. The profiles are calculated for a geographic latitude of $75^{\circ}$, a geographic longitude of $0^{\circ}$ and for a geomagnetic index $A_{p}=6$, solar activity conditions $\mathrm{F}_{10.7}=191, \overline{\mathrm{F}}_{10.7}=216$. We find that the Hall conductivity is maximum at lower altitudes than the Pedersen component, both for incident electrons and protons, and that the altitude of the maximum in $\sigma_{\mathrm{P}}$ and $\sigma_{\mathrm{H}}$ profiles decreases with increasing mean energy. A proton flux of $1 \mathrm{~mW} / \mathrm{m}^{2}$ of 
$1 \mathrm{keV}$ mean energy induces a peak in the Pedersen conductivity around $140 \mathrm{~km}$ reaching $1.2 \times 10^{-4} \mathrm{mho} \mathrm{m}^{-1}$, while at $30 \mathrm{keV}, \sigma_{\mathrm{P}}$ peaks at $130 \mathrm{~km}$ with $2 \times 10^{-4} \mathrm{mho} \mathrm{m}^{-1}$. The intensity of the Pedersen conductivity maximum does not vary as much with increasing mean energy as the maximum Hall conductivity. For electrons, with a mean energy of $1 \mathrm{keV}$, the maximum in $\sigma_{\mathrm{P}}$ occurs near $130 \mathrm{~km}$ with $8 \times 10^{-5} \mathrm{mho} \mathrm{m}^{-1}$ and in $\sigma_{\mathrm{H}}$ around $120 \mathrm{~km}$ with $7 \times 10^{-5} \mathrm{mhom}^{-1}$. For $<\mathrm{E}_{\mathrm{el}}>=4 \mathrm{keV}$, the Pedersen conductivity profiles peaks between 120 and $125 \mathrm{~km}$ and reaches $1.8 \times 10^{-4} \mathrm{mho} \mathrm{m}^{-1}$, while the Hall conductivity profile peaks around $115 \mathrm{~km}$ with a value of $3 \times 10^{-4} \mathrm{mho} \mathrm{m}^{-1}$. Finally, for a higher mean energy the maximum of Pedersen conductivity decreases while still it increases for the Hall conductivity. For example, when $<\mathrm{E}_{\mathrm{el}}>=20 \mathrm{keV}, \sigma_{\mathrm{P}} \approx 7 \times 10^{-5} \mathrm{mho} \mathrm{m}^{-1}$ around $120 \mathrm{~km}$ and $\sigma_{\mathrm{H}} \approx 3.5 \times 10^{-4} \mathrm{mho} \mathrm{m}^{-1}$ for altitudes less than $100 \mathrm{~km}$.

The Pedersen and Hall conductances are obtained by height integration of the respective conductivities between 80 and $200 \mathrm{~km}$. They are shown in Fig. 1 and Fig. 2, respectively. Figure 1 shows that the Pedersen conductance reaches a maximum for electron mean energy between 2 and $4 \mathrm{keV}$ and decreases for increasing energy, while the Hall conductance reaches a maximum for energy around $20 \mathrm{keV}$. For protons (Fig. 2), the Pedersen conductivity varies slightly between 6 and 8 mho in the energy range of $1 \mathrm{keV}$ to $40 \mathrm{keV}$. The Hall conductance increases in this energy range and reaches $9.5 \mathrm{mho}$ for $<\mathrm{E}_{\mathrm{pr}}>=40 \mathrm{keV}$.

\subsection{Conductance maps}

Hall and Pedersen conductivity height profiles and conductances are calculated for each pixel of the FUV images. We use the geomagnetic field B from the IGRF-2000 model (Olsen, 2000), evaluated separately for each pixel of each analyzed FUV image. The MSIS input parameters are adapted to the geophysical and geographic conditions of each pixel of each observation.

The EUV solar radiation also exerts an influence on the ionospheric conductances. The solar EUV radiation is the main source of ionization in the dayside ionosphere. The contribution of the EUV radiation is added in the auroral conductance maps. The determination of these empirical formulas is based on a photochemical equilibrium model of ionospheric conductivities and measurements of the Arecibo and Chatanika radars (Rasmussen et al., 1988)

$$
\begin{aligned}
& \Sigma_{P}^{\text {sun }}=\frac{4.5}{B}\left(1-0.85 v^{2}\right)\left(1+0.15 u+0.05 u^{2}\right) \\
& \Sigma_{H}^{\text {sun }}=\frac{5.6}{B}\left(1-0.9 v^{2}\right)\left(1+0.15 u+0.05 u^{2}\right),
\end{aligned}
$$

where $\nu=\chi / 90^{\circ}, \quad \mathrm{u}=\mathrm{F}_{10.7} / 90$. The solar zenith angle is in degrees, the $10.7-\mathrm{cm}$ solar flux is in units of $10^{-22} \mathrm{~W} \mathrm{~m}^{-2} \mathrm{~Hz}^{-1}$, the magnetic field strength in gauss, and the conductance in mho. These formulas are normally applicable for $0 \leq \chi \leq 85^{\circ}$ and $70 \leq \mathrm{F}_{10.7} \leq 250$ but for each pixel we extrapolate them for higher solar zenith angles.

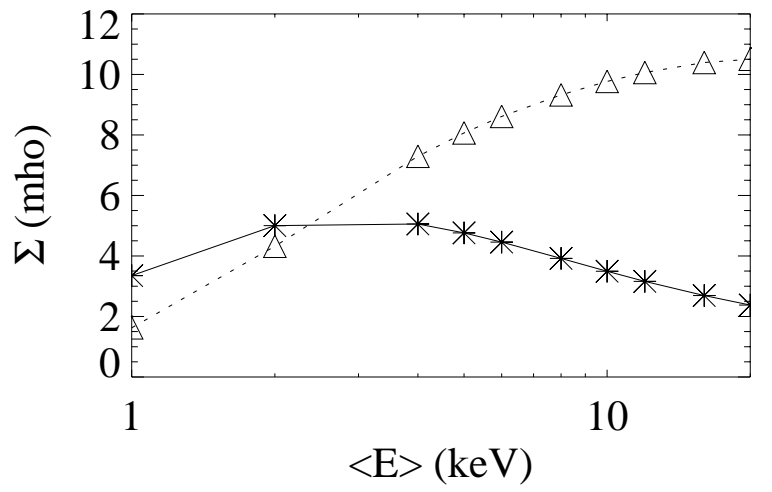

Fig. 1. Hall and Pedersen conductances due to auroral electrons. Conductances are evaluated for an incident energy flux of $1 \mathrm{~mW} / \mathrm{m}^{2}$. The Pedersen conductance is presented with stars and the Hall conductance with triangles.

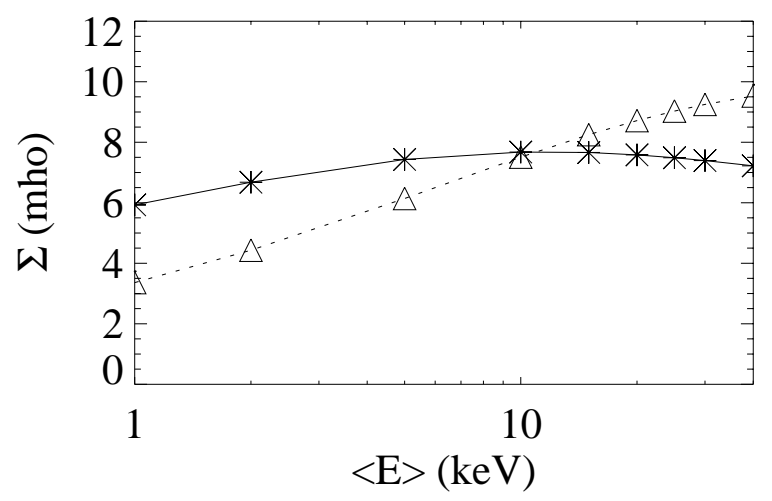

Fig. 2. Hall and Pedersen conductances due to auroral protons. Conductances are evaluated for an energy flux of $1 \mathrm{~mW} / \mathrm{m}^{2}$. The Pedersen conductance is presented with stars and the Hall conductance with triangles.

Knowing the solar zenith angle, we calculate the solarEUV contribution to the conductances and combine it with particle-induced conductances using

$\Sigma_{\text {total }}=\left(\Sigma_{\text {sun }}^{2}+\Sigma_{\text {particles }}^{2}\right)^{1 / 2}$.

The combination of two conductances produced by distinct sources is discussed in Galand and Richmond (2001a). Wallis and Budzinski (1981) estimated the error produced by this approximation to $7 \%$ for $\Sigma_{\mathrm{P}}$ and $15 \%$ for $\Sigma_{\mathrm{H}}$.

\section{Sources of uncertainties}

The results of the conductivity model we developed can be compared with results from the literature. For electrons a comparison is made with results of the Robinson et al. (1987) conductance model. Figure 3 presents the ratio of Robinson et al. (1987)'s results to the results from our model as a function of the electron mean energy. Between $3 \mathrm{keV}$ and $10 \mathrm{keV}$, differences are in a range of a few percent. Under $2 \mathrm{keV}$ 


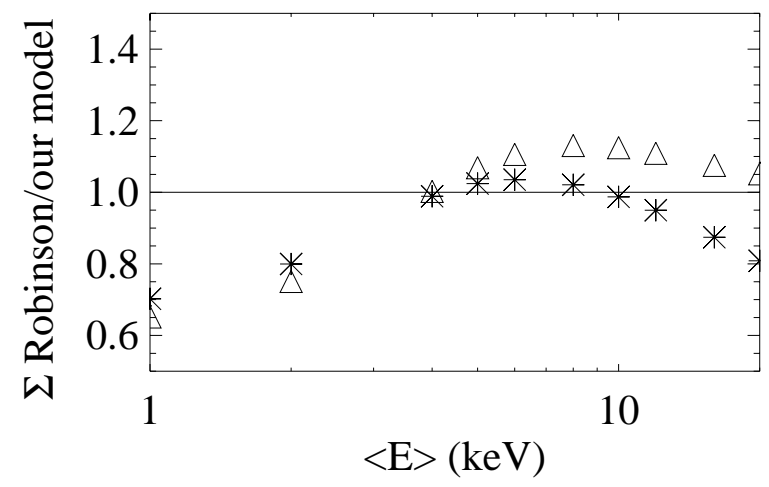

Fig. 3. Ratio between conductances due to electrons calculated with Robinson et al. (1987)'s model and values calculated with this model. The ratio for the Pedersen conductance is presented with stars and the one for the Hall conductance with triangles.

and above $10 \mathrm{keV}$ the disagreement between the models can reach $30 \%$. Statistical models of auroral electron precipitation (Hardy et al., 1985) show that the mean energy of auroral electrons is usually between 1.5 and $10 \mathrm{keV}$, where our model is quite close to Robinson et al. (1987)'s results.

Our conductance model for protons was compared with Galand et al. (2001a) using the same activity and geographic conditions. Our results are systematically 20 to $30 \%$ higher then Galand et al. (2001a). This is possibly due to the different assumptions on the distribution of the incident proton energy flux: Galand et al. (2001a) assume the distribution to be Maxwellian while we use Kappa functions, which appear to better represent the high-energy tail of protons.

\subsection{Uncertainties in the particle mean energy and energy} flux

Uncertainties are introduced by using an empirical model of particle mean energy. The empirical model cannot show individual details of the instantaneous particle precipitation. This is another potential source of error of our model of conductivity. Since the energy flux computation used the mean energy values, some error is also introduced in the conductance calculation through the energy flux. Meurant et al. (2003) evaluated the uncertainty on the electron mean energy and energy flux, comparing them with in situ measurements, when the spatial distribution of the mean energy is computed from simultaneous WIC and SI13 images. Meurant et al. (2003) showed that the precipitation characteristics derived from IMAGE-FUV data agreed with in situ measurements of two NOAA-16 passes within about $45 \%$.

To evaluate the error on the particles characteristics, the mean energy from Hardy et al. $(1985,1989)$ 's models and the energy flux deduced from FUV data were compared with in situ measurements of the FAST and DMSP satellites. Moreover, the electron mean energy computed from WIC and SI13 observations is also compared with in situ measurements and is discussed in Sect. 6. Two comparisons are described next.

\subsubsection{Comparison with DMSP in situ measurements}

Figure 4 shows an example of comparisons between the particle mean energies and energy fluxes evaluated from FUV and measured by DMSP F15 satellite for 21 October 2001. DMSP F15 is in a near circular, sun-synchronous, polar orbit. The onboard SSJ/4 detectors measure the flux of precipitating electrons and protons in the range of $32 \mathrm{eV}$ to $30 \mathrm{keV}$ in 20 channels. The total energy flux is computed by adding the flux from all channels and the mean energy is obtained by dividing the total energy flux by the total particle flux. The calculation of particle energy and flux from FUV data was described in Sect. 2. The comparisons are made after following proper smoothing of the DMSP in situ flux measurements, to account for the difference in spatial and time resolutions between in situ data and the FUV observation. Figures $4 b$ and c show, respectively, comparisons of electron mean energy and energy flux while the DMSP-F15 satellite crossed the auroral oval. Characteristics of proton precipitation are shown in Figs. 4d and e. In this example, the DMSP satellite crosses the auroral region in the pre-noon sector from 22:00 to $21: 30 \mathrm{MLT}$ and from $44^{\circ}$ to $71^{\circ}$ MLAT. Figure $4 \mathrm{a}$ shows the track of DMSP-F15 mapped to $120 \mathrm{~km}$ reported on the WIC image at 23:35:29 UT.

In Fig. 4d, the proton mean energies measured by DMSP present a maximum around $25 \mathrm{keV}$ between 23:36:50 and 23:37:00 UT. The values are quite constant, around $15 \mathrm{keV}$, between 23:37:00 and 23:39:40 UT. After 23:39:40 UT the proton mean energy values reach $30 \mathrm{keV}$. The mean energies from Hardy et al. (1989)'s model are in the same range of value but do not show two maxima. When the measured mean energy is about $15 \mathrm{keV}$ around 23:38:20 UT, the empirical model overestimates it and indicates a maximum energy of $25 \mathrm{keV}$. The disagreement between the empirical model and the in situ measurements in this region is $66 \%$. The comparison of the proton energy flux measured by DMSP satellite and evaluated from the Hardy et al. (1989) mean energy and SI12 observations is plotted in Fig. 4e. The maximum in the flux computed from FUV data is shifted in comparison with the measurements. Moreover, the measured energy flux is overestimated during the time range 23:37:30 and 23:39:20 UT. This is probably due in part to the difference of spatial and time resolutions of instruments, and in part to the disagreement between used energy and real energy in this time range. The global agreement between the energy fluxes measured in situ and evaluated from FUV observation is $\sim 19 \%$.

For electrons, Fig. 4b shows that the Hardy et al. (1985) statistical mean energies are in good agreement with the DMSP measurements. The values are in the same range: around 0 and $2 \mathrm{keV}$ between 23:36:00 and 23:38:20 and between $2 \mathrm{keV}$ and $5 \mathrm{keV}$ after. Around 23:39:50 and 23:40:40 measurements show, respectively, a minimum and a maximum in the energy that the statistical model cannot present. In Fig. $4 c$ the energy fluxes computed from the statistical mean energy and the WIC data underestimate the DMSP energy flux between 23:38:10 and 23:39:30. In this case, 
(a)
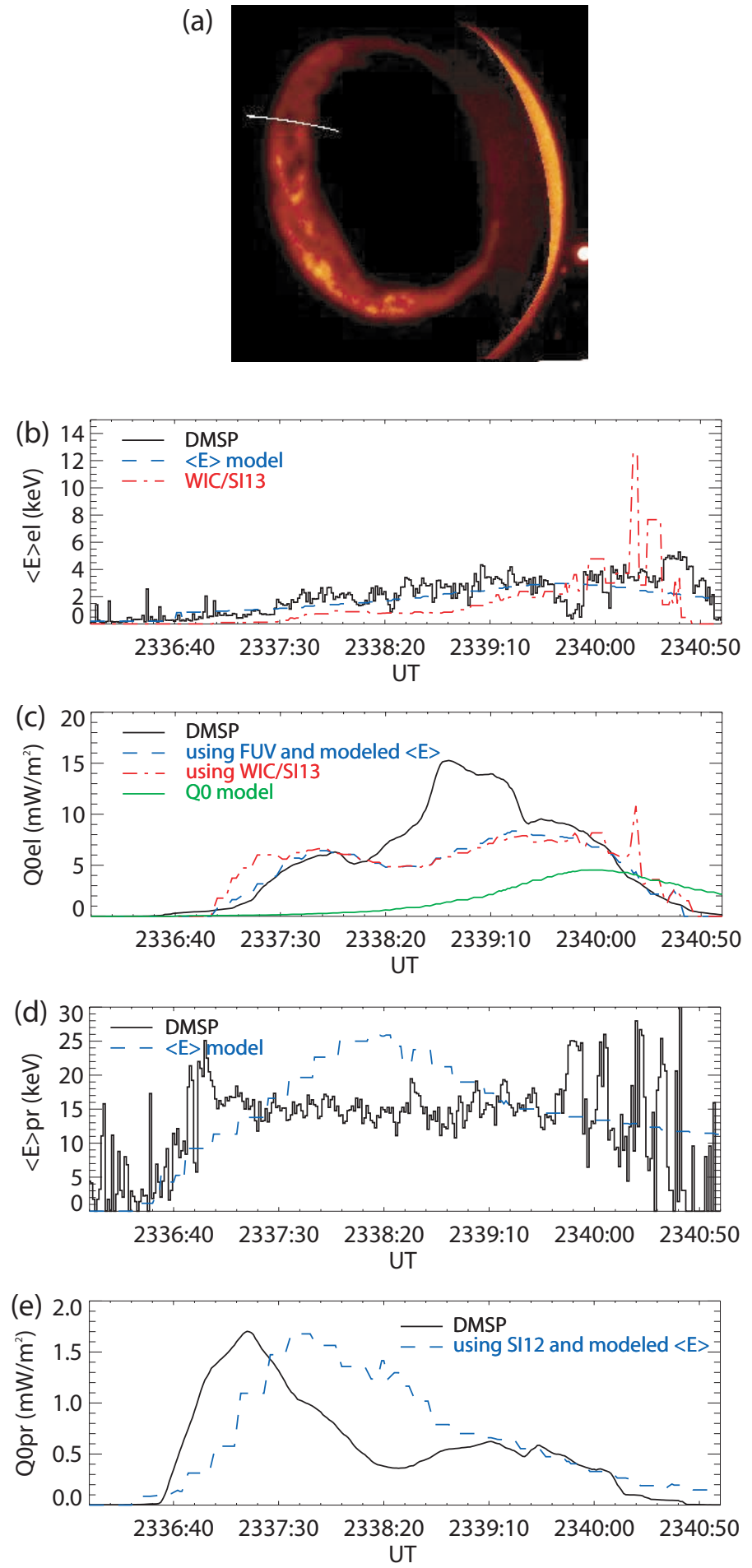

Fig. 4. Comparison between the particle precipitation characteristics evaluated from the FUV data and measured by the DMSP F15 satellite for 21 October 2001. (a) The mapped position of the DMSP F15 satellite is drawn on the WIC image. (b) The electron mean energy from Hardy et al. (1985) (blue curve) is compared with mean energy in situ measurement (black curve) and with mean energy evaluated from the ratio between WIC and SI13 images (red curve). (c) The electron energy flux evaluated using FUV and Hardy et al. (1985) mean energy (blue curve) and the one evaluated using the mean energy from WIC/SI13 ratio (red curve) are compared with DMSP energy flux measurement (black curve) and with Hardy et al. (1985) electron energy flux (green curve). (d) The proton mean energy from Hardy et al. (1991) (blue curve) is compared with the DMSP proton mean energy (black curve). (e) The energy flux evaluated from FUV-SI12 and the Hardy et al. (1991) mean energy (blue curve) is compared with in situ measurement (black curve). 
(a)
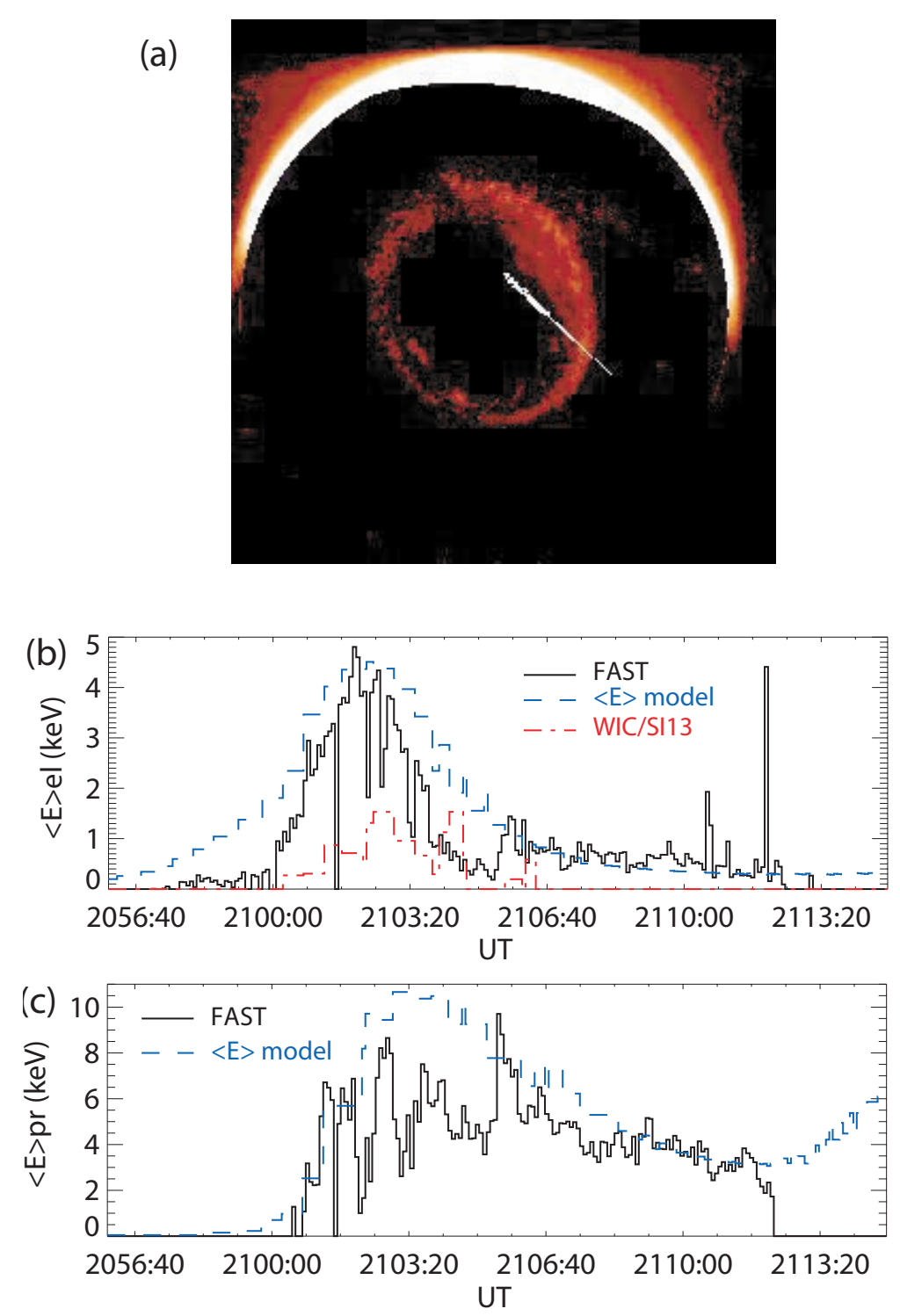

Fig. 5. Comparison between the particle precipitation characteristics evaluated from the FUV data and measured by the FAST satellite for 23 December 2000. (a) The mapped position of the FAST satellite is drawn on the WIC image. (b) The electron mean energy from the Hardy et al. (1985) empirical model (blue curve) is compared with mean energy in situ measurement (black curve) and with mean energy evaluated from the ratio between WIC and SI13 images (red curve). (c) The proton mean energy from Hardy et al. (1991) (blue curve) is compared with the FAST proton mean energy (black curve).

the error is a factor of 2. The agreement is good elsewhere along the trajectory. The dash-dot line in Fig. $4 \mathrm{c}$ shows the electron energy flux from Hardy et al. (1985)'s model. The morphology of the precipitation is very different when using the statistical model's outputs. The statistical flux is nearly null until 23:38:10 UT, while DMSP measurements show that it reaches a first maximum at $6 \mathrm{~mW} / \mathrm{m}^{2}$ around 23:38:00 UT. The statistical flux increases up to $5 \mathrm{~mW} / \mathrm{m}^{2}$ at 23:40:00 and then decreases while in situ measurements peaks up to $15 \mathrm{~mW} / \mathrm{m}^{2}$ at 23:38:50 UT and decreases down to 0 at 23:41:00 UT. This comparison shows that the Hardy et al. (1985) energy flux model is not a good estimation of the instantaneous energy flux.

\subsubsection{Comparison with FAST in situ measurements}

In a second step, we compare the mean energy measurements determined from the FAST satellite detectors with the statistical model outputs for 23 December 2000. Figures $5 b$ and c present the comparison for electron and for proton mean energies, respectively. In this example, the FAST satellite crosses the auroral oval in the dawn sector from 04:18 to 08:30 MLT between $57^{\circ}$ and $81^{\circ}$ MLAT. Figure 5a shows the track of the FAST satellite mapped to $120 \mathrm{~km}$ traced on the WIC image obtained at 21:00 UT. 
The comparison of the electron mean energy (Fig. 5b) shows that the agreement between the Hardy et al. (1989)'s model mean energies and the in situ measurements is very good. The electron mean energies measured by FAST and computed from the statistical model follow the same evolution. Around 21:02:30 UT the maximum values in both measured and modeled energies are about $4.5 \mathrm{keV}$. In the region crossed after 21:05:50 UT, both energies are under $1 \mathrm{keV}$, even if the measurements show some very local peaks. Globally, the statistical energy is 35\% higher than the FAST measurement, even if, very locally, the disagreement can reach up to $100 \%$.

For protons, Fig. 5c shows that globally the empirical model and the measurements are in the same range. Around 21:03:20 UT the statistical mean energy reaches $11 \mathrm{keV}$, while the in situ measurements are equal to $7 \mathrm{keV}$. After 21:12:30 UT the measured energy is null while the statistical energy increases. However, this is of no consequence for the conductance calculation, because the associated energy flux is negligible. The final conductance is very low, so that the relative error is not so important.

\subsubsection{Uncertainties in the conductance model}

We now try to evaluate the error on the conductance calculation introduced by the uncertainties on the particle mean energy. For electrons, we noted in Sect. 4.1.2 that the error can be locally very important. As an example, we consider an error of 50\% and calculate the propagated error on conductance. Figure 1 indicates that an error of $\pm 50 \%$ at $1 \mathrm{keV}$ induces an error of $+33 \%$ or $-57 \%$ on the Pedersen conductance, and $+89 \%$ or $-76 \%$ on the Hall conductance evaluation. If the electron mean energy is $5 \mathrm{keV}$, the error is $-15 \%$ and $+9 \%$ for Pedersen conductance, and $+14 \%$ and $-34 \%$ for Hall conductance. At $10 \mathrm{keV}$, the error is between $-20 \%$ and $+36 \%$ for Pedersen conductance and $+6 \%$ and $-17 \%$ for Hall conductance.

The error on the proton mean energy evaluated from comparison between in situ measurements and the statistical model outputs for the cases described before is around $65 \%$. Using Fig. 2, it is possible to evaluate the error introduced by the method. If we use $10 \mathrm{keV}$ while the real mean energy is $30 \mathrm{keV}$, the Pedersen conductance is overestimated by $4 \%$ and the Hall conductance underestimated by $19 \%$. Using $50 \mathrm{keV}$ for calculation while the proton mean energy is $30 \mathrm{keV}$ leads to an underestimation of the Pedersen conductance by $4 \%$ and an overestimation of the Hall conductance by $5 \%$. For energy around $30 \mathrm{keV}$ an error of $66 \%$ in the mean energy introduces an error of $19 \%$ at most in the conductance determination. For lower energies, the relative error is higher. For example, using $\left.<\mathrm{E}_{\mathrm{P}}\right\rangle=1 \mathrm{keV}$ in calculation while the proton mean energy is $3 \mathrm{keV}$ introduces a $16 \%$ error in the Pedersen conductance and a 35\% error in the calculated Hall conductance. Generally, the uncertainty in the particle mean energy leads to a larger error in the Hall conductance determination than in the Pedersen conductance, both for electrons and protons.
An uncertainty in the particle mean energy introduces an error in the energy flux which can influence the conductance estimation. As we discussed in the comparison with DMSP measurements (Sect. 4.1.1) the error in the proton energy flux is around 19\%. In the comparison in Sect. 4.1.1 the discrepancy is locally more important.

\subsection{Influence of geomagnetic activity}

The particle energy transport models uses a MSIS-90 (Hedin, 1991) atmosphere with fixed geomagnetic and activity level conditions. The MSIS parameters for 24 December 2000, 16:30 UT, a latitude of $65^{\circ}$, a longitude of $0^{\circ}$, solar maximum conditions with $\mathrm{F}_{10.7}=205, \overline{\mathrm{F}}_{10.7}=216$, and a geomagnetic index $A_{p}=6$. We now analyze the influence on the conductance evaluation of the use of fixed atmospheric conditions. For protons, the Monte Carlo code was run for four different situations encompassing a range of solar activity conditions: (i) $A_{p}=5, \mathrm{~F}_{10.7}=\overline{\mathrm{F}}_{10.7}=200$, (ii) $A_{p}=40, \mathrm{~F}_{10.7}=\overline{\mathrm{F}}_{10.7}=200$, (iii) $A_{p}=5, \mathrm{~F}_{10.7}=\overline{\mathrm{F}}_{10.7}=80$, and (iv) $A_{p}=20, \mathrm{~F}_{10.7}=\overline{\mathrm{F}}_{10.7}=80$. The other parameters were fixed to 24 December 2000, 00:00 UT, a latitude of $60^{\circ}$, a longitude of $0^{\circ}$ for a monoenergetic proton incident beam of $1 \mathrm{~mW} / \mathrm{m}^{2}$ with an energy of $10 \mathrm{keV}$. Cases (i) and (ii) present solar maximum conditions while cases (iii) and (iv) are characteristic of the solar minimum activity. Using condition of case (i), the calculated conductances were: $\Sigma_{\mathrm{P}}=8.71 \mathrm{mho}$ and $\Sigma_{\mathrm{H}}=5.74 \mathrm{mho}$. An increase in the $A_{p}$ index for solar maximum conditions (case (ii)) gives $\Sigma_{\mathrm{P}}=8.63 \mathrm{mho}$ and $\Sigma_{\mathrm{H}}=5.13$ mho which means a decrease of $0.8 \%$ for the Pedersen conductance and of $10 \%$ for the Hall conductance. An increase in the $A_{p}$ index for solar minimum conditions (case (iv) compared with case (iii)) induces, respectively, an increase in $0.3 \%$ and a decrease of $7.8 \%$ in the Pedersen and Hall conductances. A change in the $\mathrm{F}_{10.7}$ index (case (iii) compared with case (i)) produces a variation of $5.1 \%$ for Pedersen conductance and $11.6 \%$ for Hall conductance. We conclude that for protons the variation of solar and geomagnetic activity introduces effects of $\sim 5 \%$ and $12 \%$ for Pedersen and Hall conductances. For electrons a similar study shows that the maximum uncertainties associated with the $A_{p}$ index and/or the $\mathrm{F}_{10.7}$ index is $\sim 11 \%$ for the Pedersen conductance and around $2 \%$ for the Hall conductance.

This discussion presented the uncertainty sources that appear in our method of conductance calculation. The use of averaged solar activity and geomagnetic conditions induced a maximal error of $12 \%$ while the uncertainty in the particle mean energy can induce a more important error. This is the main source of error in the conductance calculation. In Sect. 6, we describe another method to obtain the instantaneous electron mean energy and we discuss the results. 


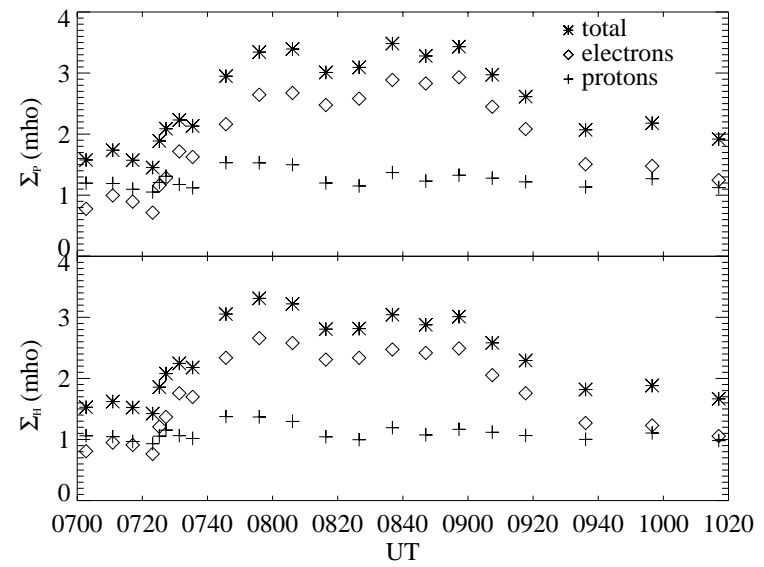

Fig. 6. Time evolution of the Pedersen (top panel) and Hall (bottom panel) conductances during a substorm which occurred on 27 November 2000. The conductances are averaged over all the auroral region.

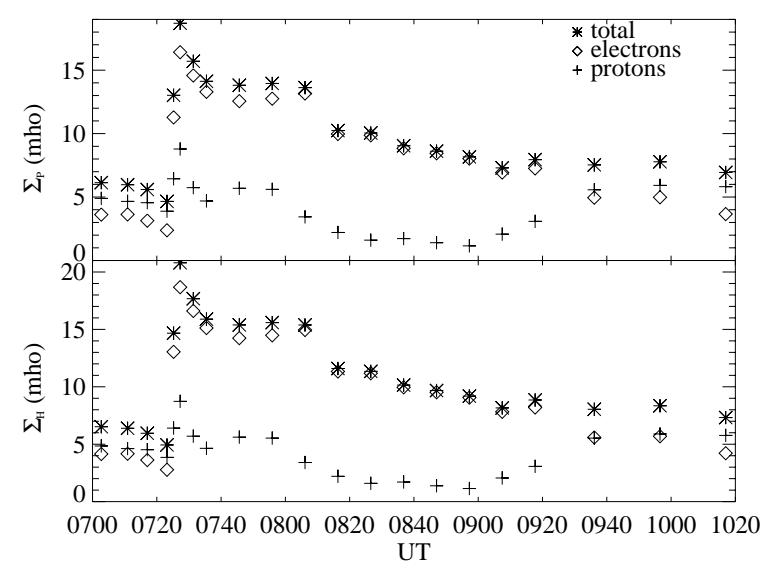

Fig. 7. Idem Fig. 6. In this example, the conductances are averaged over the midnight sector (MLAT between $60^{\circ}$ and $65^{\circ}$ and MLT between 23:30 and 00:30).

\section{Global conductance maps}

In this section, we present 4 selected cases with different $K_{p}$ index values, observed in the Northern Hemisphere. We illustrate different auroral activity levels at different seasons. We first present instantaneous conductance maps calculated for 12 August 2000 and 23 December 2000. The specificity of this work is that instantaneous conductance maps are evaluated each $2 \mathrm{~min}$ so that the evolution of conductances during substorms can be followed. It is, thus, possible to assess the evolution of the role of protons in the conductances. In a second step we present the evolution of conductances in different magnetic sectors during two selected substorms which occurred on 27 November 2000 and 28 April 2001. For each case and for both conductances, we calculate the increase in conductance when protons are considered in comparison with a pure electron precipitation assumption.

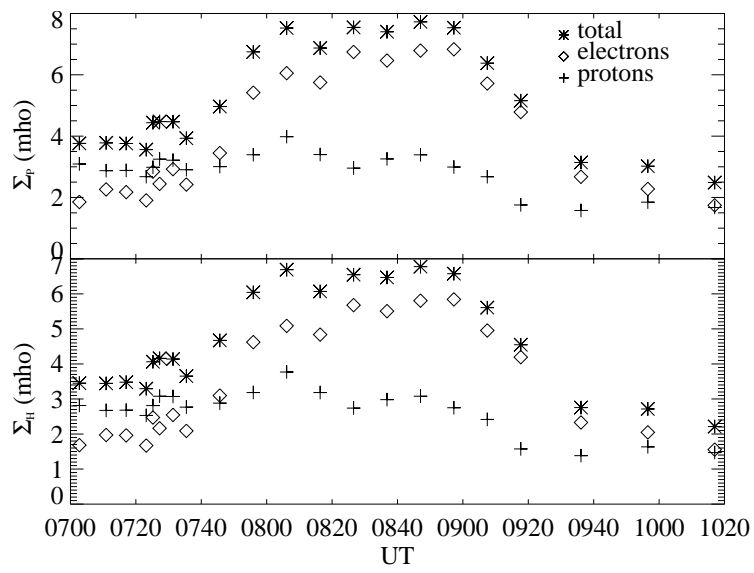

Fig. 8. Idem Fig. 6 with conductances averaged over the dusk sector (MLAT between $60^{\circ}$ and $70^{\circ}$ and MLT between 18:00 and 21:00).

\subsection{August 2000}

The first example shown in Plate 1 occurred during the expansion phase of a summer substorm $\left(K_{p}=8\right)$ on 12 August 2000 at 08:32 UT. The top panels show the calculated Pedersen (Plate 1a) and Hall (Plate 1b) conductances induced by auroral electron precipitation. Central panels show Pedersen (Plate 1c) and Hall (Plate 1d) conductances induced by protons. Bottom panel (Plate 1e and f) shows conductance maps induced by auroral particles and the solar EUV contribution. Adding the proton contribution in the conductance calculation globally increases the Hall conductance by $5.5 \%$ and the Pedersen conductance by $4.8 \%$ in comparison with the calculation for electrons only. In the sector between 21:00 and 24:00 MLT, the proton contribution is $\sim 8 \%$ for $\Sigma_{\mathrm{P}}$ and $\Sigma_{\mathrm{H}}$, while in the post-noon sector protons contribute less than $1 \%$ in both $\Sigma_{\mathrm{P}}$ and $\Sigma_{\mathrm{H}}$. The EUV contribution to the conductances can be more important than the proton contribution during this summer substorm. The maximum in the EUV contribution to the Hall conductance near 12:00 MLT and $55^{\circ}$ MLAT is $\sim 13 \mathrm{mho}$, while the maximum in the Hall conductance due to protons is less than 6 mho. The Hall conductance can locally reach 40 mho and the Pedersen conductance $\sim 28$ mho.

\subsection{December 2000}

The second example (Plate 2 ) is a winter situation with a low magnetic activity level $\left(K_{p}=2\right)$ which occurred on 23 December 2000 at 21:04 UT. It corresponds to a quiet phase between two weak substorms. The particle mean energies were locally validated by comparison with FAST in situ measurements (described in Sect. 4.1.2). A feature of this case, fairly typical of a non-substorm situation, is that the proton contribution to both Hall and Pedersen conductances is maximum in the dusk sector, whereas the electron contribution is close to zero. The maximum in the electron induced conductances is located near 07:00 MLT in the dawn sector. Electron precipitation is relatively important in the entire pre-noon sector. 

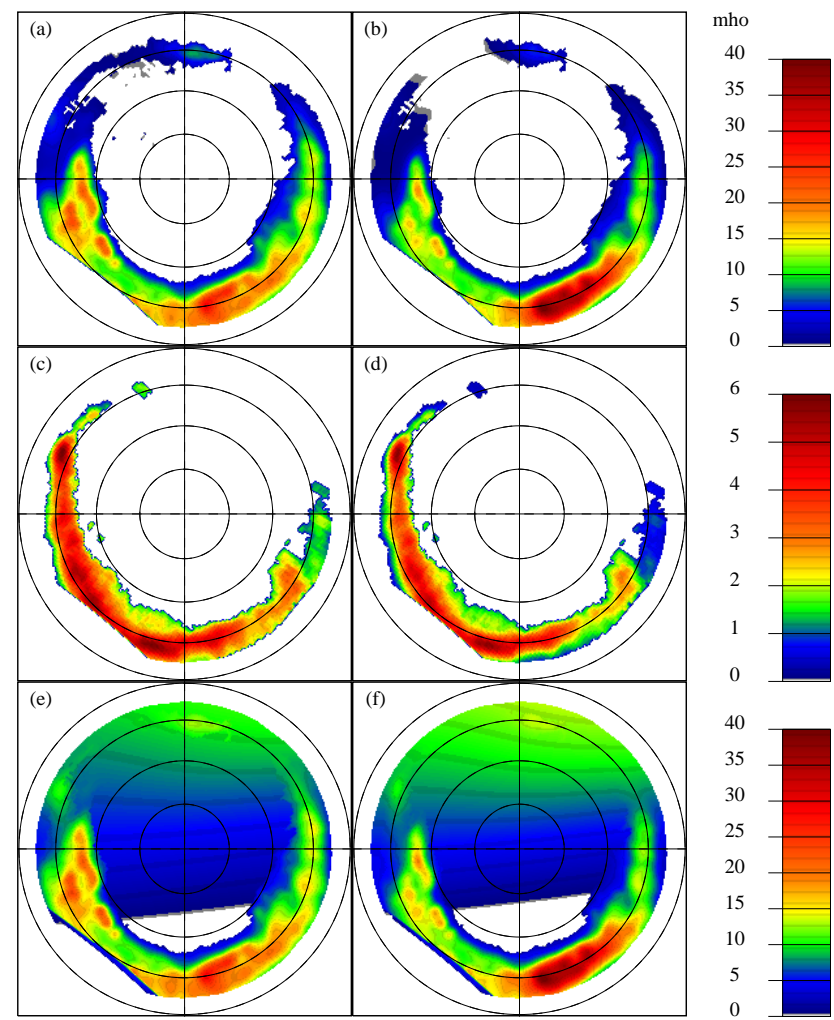

Plate 1. Conductance maps for 12 August 2000, 08:32 UT corresponding to a summer substorm $\left(K_{p}=8\right)$. Panels (a) and (b) show, respectively, Pedersen and Hall conductances induced by auroral electron precipitation. Panels (c) and (d) show, respectively, Pedersen and Hall conductances induced by protons and panel (e) and (f) show conductance maps induced by both auroral particles and EUV solar radiation. Circles show $50^{\circ}, 60^{\circ}, 70^{\circ}$ and $80^{\circ}$ MLAT. The local midnight is located downward.

The maximum in the Hall and Pedersen conductances induced by electrons are 8.2 mho and 5.3 mho, respectively, while the maximum in conductance induced by protons are 3.1 mho and 3 mho, respectively. We note that the conductance values are small in this case. Globally, over the entire auroral oval, the protons increase the Hall conductance by $40 \%$ and the Pedersen conductance by $53 \%$, mostly as a consequence of the effect of proton precipitation in the dusk sector. In comparison to the substorm case discussed before, the proton contribution in conductances is globally significantly more important.

Another example (not shown) of a quiet winter situation corresponding to 1 November 2000 at 17:01 UT has been analyzed. The $K_{p}$ index for this period was equal to 2 . In this example, the auroral injection is close to zero in the dayside and weak in the nightside. The maximum in the Pedersen conductance map due to auroral particles is 7.0 mho and 7.3 mho in the Hall conductance map. Adding the contribution of proton in the conductance calculation globally increases the Hall and the Pedersen conductances by $13 \%$ in comparison with the calculation with electrons only.
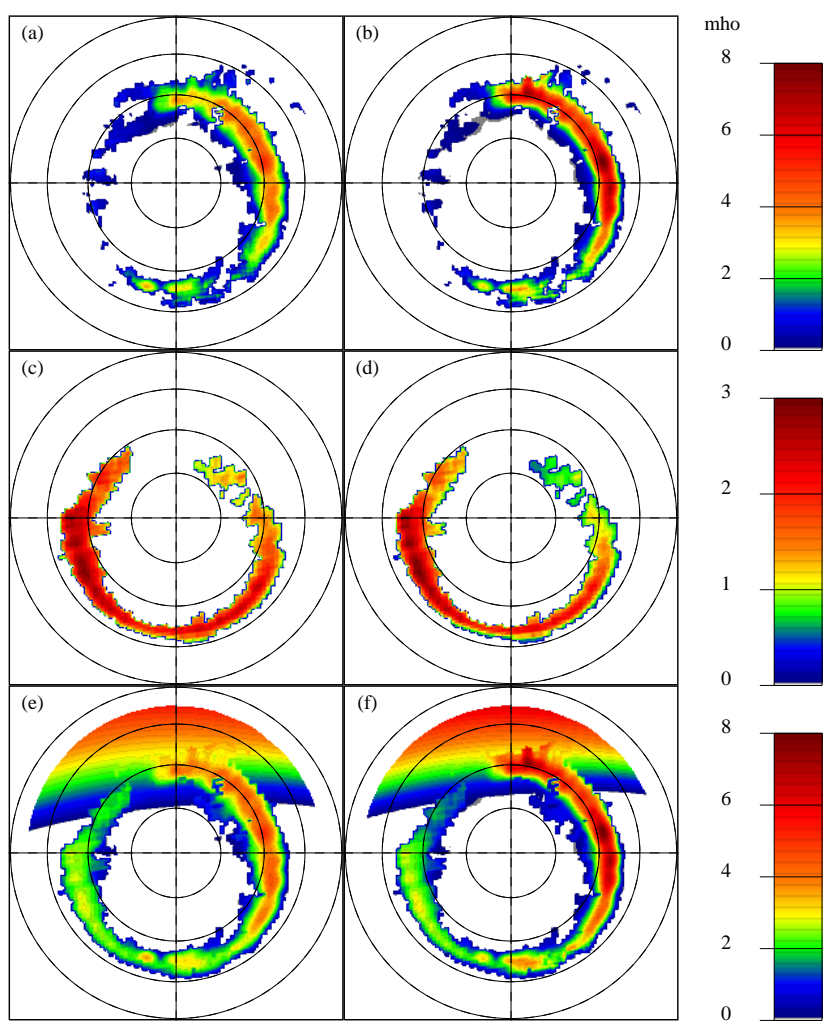

Plate 2. Idem Plate 1 for 23 December 2000, 21:04 UT with $K_{p}=2$. Circles show $50^{\circ}, 60^{\circ}, 70^{\circ}$ and $80^{\circ}$ MLAT. The local midnight is located downward.

During periods of quiet magnetic activity the relative contribution of auroral protons in conductances can be much more important than during active period. As an example, during the substorm of 12 August 2000 their contribution reached a few percent, while in the low $K_{p}$ example, it can reach more than $50 \%$ and it is even close to $100 \%$ locally. This result reflects that the relative contribution of the protons to the auroral hemispheric power is larger during quiet time periods.

To test the sensitivity of the method, conductance maps were constructed for the case of 23 December 2000 using a constant particle mean energies for all FUV pixels instead of the statistical mean energies. Plate 3 shows the result using $<\mathrm{E}>_{\mathrm{el}}=6.4 \mathrm{keV}$ and $<\mathrm{E}>_{\mathrm{pr}}=8 \mathrm{keV}$. The value for electron is chosen as the maximal value of the pre-noon sector average energy for $K_{p}=3$ in the Hardy et al. (1987)'s empirical precipitation model. For protons a mean energy of $8 \mathrm{keV}$ is a plausible value for low activity conditions according to Hardy et al. (1991). The results (Plate 3b) show an increase in the Hall conductance maximum of about $20 \%$ for the electron contribution when using $6.4 \mathrm{keV}$ instead of the statistical average energy (Plate 2b). On the poleward limit of the auroral zone in the morning sector, the Hall conductance value is around 5 or 6 mho, while using the statistical mean energies the values are around 1 mho. Thus, when the constant energy case is very different from the statistical energy, the differences in the Hall conductance computation can be 


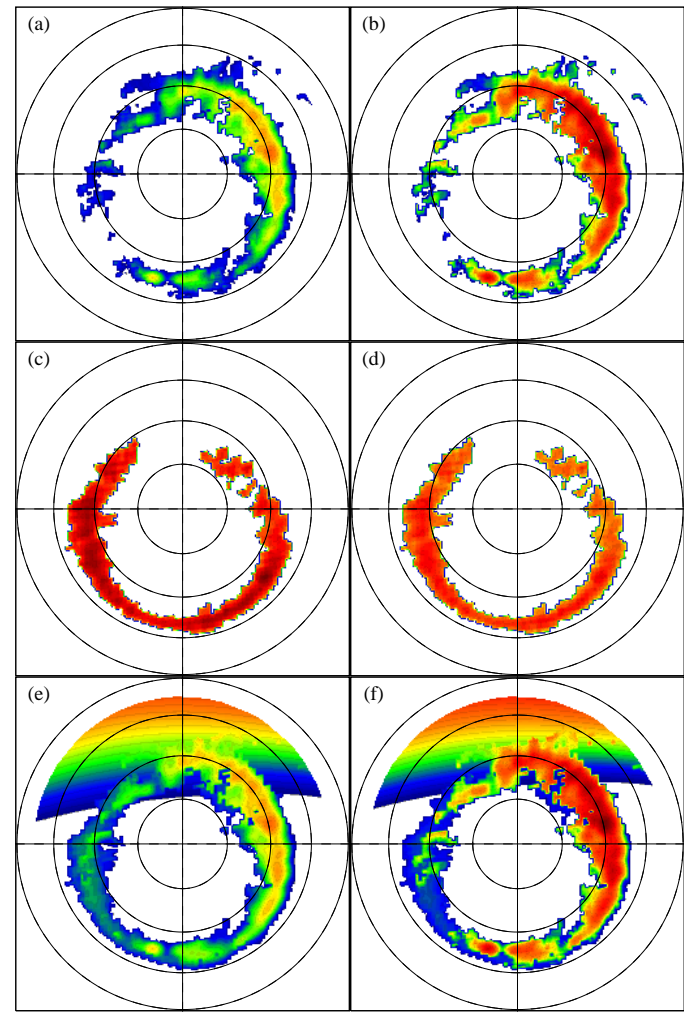

mho

10

8

6

4

\section{2}

0

2

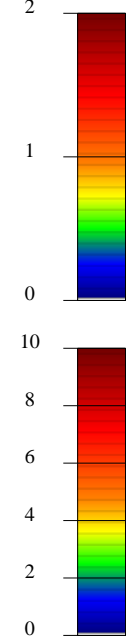

Plate 3. Conductance maps for 23 December 2000, 21:04 UT (same as Plate 2) using constant particle mean energy over the entire auroral region. $<\mathrm{E}>{ }_{\mathrm{el}}=6.4 \mathrm{keV}$ and $<\mathrm{E}>{ }_{\mathrm{pr}}=8 \mathrm{keV}$. Circles show $50^{\circ}$, $60^{\circ}, 70^{\circ}$ and $80^{\circ}$ MLAT. The local midnight is located downward.

locally very important. This effect was discussed in Sect. 4.1. The effects on the Pedersen conductance computation are much less significant. The maximum in Pedersen conductance (Plate 3a) has a value nearly identical using statistical average energy (Plate 2a) and the morphology of the induced conductance is similar. The calculation of conductances induced by proton precipitation with a constant mean energy is presented in Plates $3 \mathrm{c}$ and $\mathrm{d}$. Comparing with Plates 2c and $\mathrm{d}$, the conductances values globally decrease when using $<\mathrm{E}>_{\text {pr }}=8 \mathrm{keV}$ and the morphology of the pattern also exhibits some variations. With the statistical mean energy, the maximum in both conductance patterns is near 20:00 MLT and $65.5^{\circ} \mathrm{MLAT}$. With the constant energy, this value decreases by $46 \%$ for Pedersen and $30 \%$ for Hall conductance. Moreover, the location of the maximum in the conductance is changed. When using the constant energy, the peak in conductances is around 03:25 MLT and 65.4 $4^{\circ}$ MLAT. As already mentioned in Sect. 4.1, the uncertainties on the mean energy is the main source of errors of the method and can induce important errors. Globally, the conductance values are close to each other and the morphology is quite similar for both cases.

\subsection{November 2000}

A specific advantage of this approach is that the evolution of conductances during substorms can be analyzed. On 27 November 2000, two substorms followed each other: the first one started around 03:55 UT and the second one around 07:25 UT. The events were described by Hubert et al. (2002), who calculated the evolution of the hemispheric power and related the auroral events to solar wind variations detected by the ACE satellite. We consider the second substorm only. The onset was observed by the three FUV instruments at $\sim 07: 25$ UT near 00:00 MLT. It is related to a sudden increase in the solar wind density. The WIC observations show that for electrons, the substorm expansion extended both duskward and dawnward. The proton aurora observed by SI12 predominantly extended dawnward with a less significant duskward extension. Hubert et al. (2002) showed that the contribution of proton in the hemispheric power reached $45 \%$ before the substorm onset, decreasing under $15 \%$ after breakup. The minimum in the proton contribution took place around 08:30 UT when the total hemispheric power was maximum. The instantaneous Hall and Pedersen conductance maps were evaluated each 2 or 4 min before the onset and during the beginning of the substorm and then each 10 or 20 min during the substorm development. The evolution of conductances averaged in different MLAT-MLT sectors are illustrated in Figs. 6, 7 and 8. The time evolution of the average conductances was calculated over the entire north polar region (Fig. 6). It presents two main peaks: a first one between 07:55 and 08:06 UT and a second one around 08:36 UT which is as important as the first one for Pedersen conductance, reaching 3.6 mho, while for Hall conductance the first maximum was the highest, reaching 3.3 mho. The contribution of electrons and protons is $\sim 1$ mho for both conductances before the visually estimated onset ( $\sim 07: 25 \mathrm{UT})$. The calculation of conductances including protons is $60 \%$ higher than using electrons only, before the onset. It decreases to $\sim 20 \%$ during the substorm. After 09:00 UT, conductances due to electrons decreased while the value of the ratio $\left(\Sigma_{\text {aur }}-\Sigma_{\mathrm{el}}\right) / \Sigma_{\mathrm{el}}$ increased. In the sector between 23:30 and 00:30 MLT and $60^{\circ}$ and $65^{\circ}$ MLAT (Fig. 7), the increase in conductances due to electron and proton around 07:25 UT corresponds to the substorm onset which was visually identified from the FUV observations. The averaged Pedersen conductance due to auroral particles reaches 19.5 mho in that sector and averaged Hall conductance around 21 mho. Between $\sim 07: 00$ and $\sim 07: 11$ UT, the contributions of both conductances remain quite constant. Between $~ 07: 11 \mathrm{UT}$ and $\sim 07: 25$ UT conductances in this sector decrease and reach a minimum just before the onset. At that time the contribution of proton in conductances is maximum. Before $\sim 07: 11 \mathrm{UT}$, including the protons in conductances, evaluation increases the Pedersen conductance by about $65 \%$ and the Hall conductance by about $55 \%$. Just before the substorm onset, the Pedersen conductance increases by about $95 \%$ when adding the proton contribution, and the Hall conductance by about $80 \%$. After 07:25 UT, the proton precipitation increase both 
conductances by $\sim 10 \%$ and after 08:00 UT this contribution decreases below 5\%. Figure 8 shows the evolution of conductances in the dusk and night sector. The electron induced conductances start increasing at 07:46 UT when the substorm activity reaches that sector. Before 07:46 UT the increase in both conductances due to protons is between 100 and 50\% but it decreases under 30\% after 07:46 UT and under $20 \%$ after 08:20 UT.

\subsection{April 2001}

The example of 28 April 2001 illustrates a particular feature. Before 05:00 UT the measurements of the FUV instruments were close to their sensitivity threshold. Auroral activity increased after 05:00 UT and SI12 imaged a sudden brightening in the post-noon sector. This signal was also observed by WIC and much less intensely by SI13. At 05:08 UT a very bright spot was observed by SI12 at $\sim 74^{\circ}$ MLAT and 14:15 MLT, its luminosity gradually decreased and at 05:12 UT the spot disappeared. At 05:21 UT the spot reappeared in the SI12 images at the same location in the same time as the auroral signal intensified near 00:00 MLT. Two minutes later, the spot had vanished while the midnight sector activity was still strong. A spot was also observed with the WIC and SI13 instruments at the same location and at the same time. In view of the intensity of the signal observed by SI12, it appears that the spot in the post-noon sector is essentially due to protons. The evaluation of conductance maps from FUV observations at 05:08 UT shows that the maximum of the Pedersen and Hall conductances induced by protons occurred in the post-noon sector and reached 7.7 mho and 5.9 mho, respectively, while the maximum in the Pedersen conductance due to electron occurs around 19:30 MLT and $73^{\circ}$ MLAT and reached 8 mho (5 mho for Hall conductance at the same location). The evolution of conductances in the post-noon sector is shown in Fig. 9. The increase in both conductances induced by protons at 05:00 UT coincides with the onset of the substorm. The conductance due to protons averaged over the sector between $71^{\circ}$ and $77^{\circ}$ MLAT and 14:00 and 14:30 MLT peaked a first time at 05:08 UT, up to 5.6 mho for Pedersen and 4 mho for Hall conductance and a second time at 05:21 UT, up to 6 mho for Pedersen and 4.6 mho for Hall conductances. After 05:21 UT, the Pedersen and Hall proton induced conductances are around 2 and 1.5 mho, respectively. During the event, the averaged Pedersen and Hall conductances due to electrons in the post-noon sector remained quite constant, respectively, around $0.6 \mathrm{mho}$ and 0.2 mho. The total value of conductances due to both types of particles is very close to the value of the proton induced component in the post-noon sector. This clearly illustrates that neglecting protons in this particular case would induce a very large error in conductances evaluation in this sector.

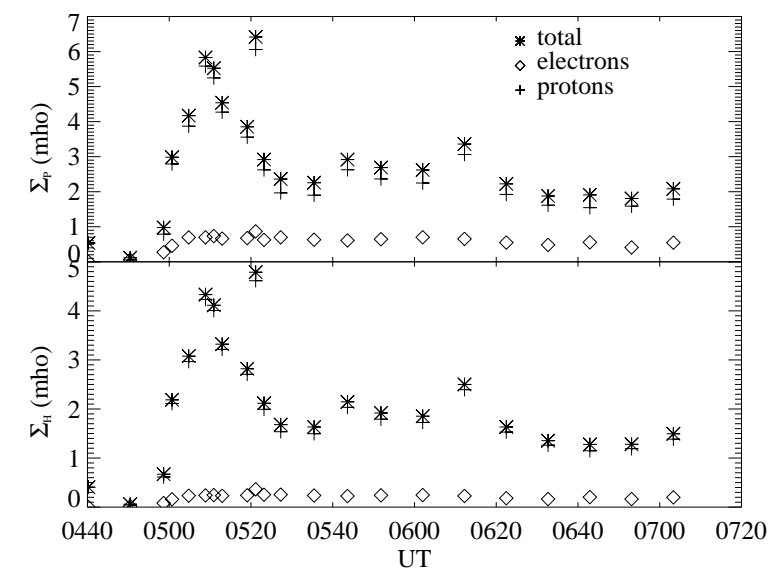

Fig. 9. Time evolution of the Pedersen (top panel) and Hall (bottom panel) conductances during a substorm which occurred on 28 April 2001. The conductances are averaged over the sector between $71^{\circ}$ and $77^{\circ}$ MLAT and 14:00 and 14:30 MLT.

\section{Electron mean energy from the WIC to SI13 ratio}

Using the electron transport models described in Sect. 2, the electron mean energy can be derived from WIC and SI13 observations. The method to determine the mean electron energy and energy flux characteristics from FUV observations and the uncertainties associated with this method were thoroughly described by Hubert et al. (2002) and Meurant et al. (2003). The determination of the electron mean energy is based on the ratio between WIC and SI13 images. The WIC/SI13 ratio was modeled as a function of the energy of the precipitating electrons using the GLOW model (Solomon et al., 1988) extended to higher energies for auroral calculations (Hubert et al., 2001).

In the calculation of conductance maps, the Hardy et al. (1985) electron mean energy can be replaced by the mean energy evaluated from the WIC to SI13 ratio. An upper threshold of the electron mean energy was fixed to $15 \mathrm{keV}$. Uncertainties are associated with this method. Meurant et al. (2003) compared the electron energy determined using the WIC/SI13 ratio with the electron energy measured by the detectors on board the NOAA-16 satellite and found agreement within about $45 \%$. In addition, we compared the FUV derived electron mean energy with the DMSP and FAST in situ measurements. In Fig. $4 \mathrm{~b}$ the electron mean energy deduced from the WIC/SI13 ratio is compared with the Hardy et al. (1985) model and DMSP measurement. In the region between 23:36:00 and 23:39:40 UT the electron mean energy from the WIC/SI13 ratio underestimates the in situ measurement. Between 23:37:30 and 23:39:40 UT the disagreement is about $50 \%$ or more. After 23:40:00 UT the FUV extracted energy presents two maxima: a first one peaks up to $12.5 \mathrm{keV}$ and the second one up to $7.5 \mathrm{keV}$, which are not measured by DMSP. Such peaks are generally induced by the background signal subtraction in the WIC data. However, in this case, DMSP crossed the auroral oval in the night sector. The WIC signal is thus not contaminated by dayglow signal. 

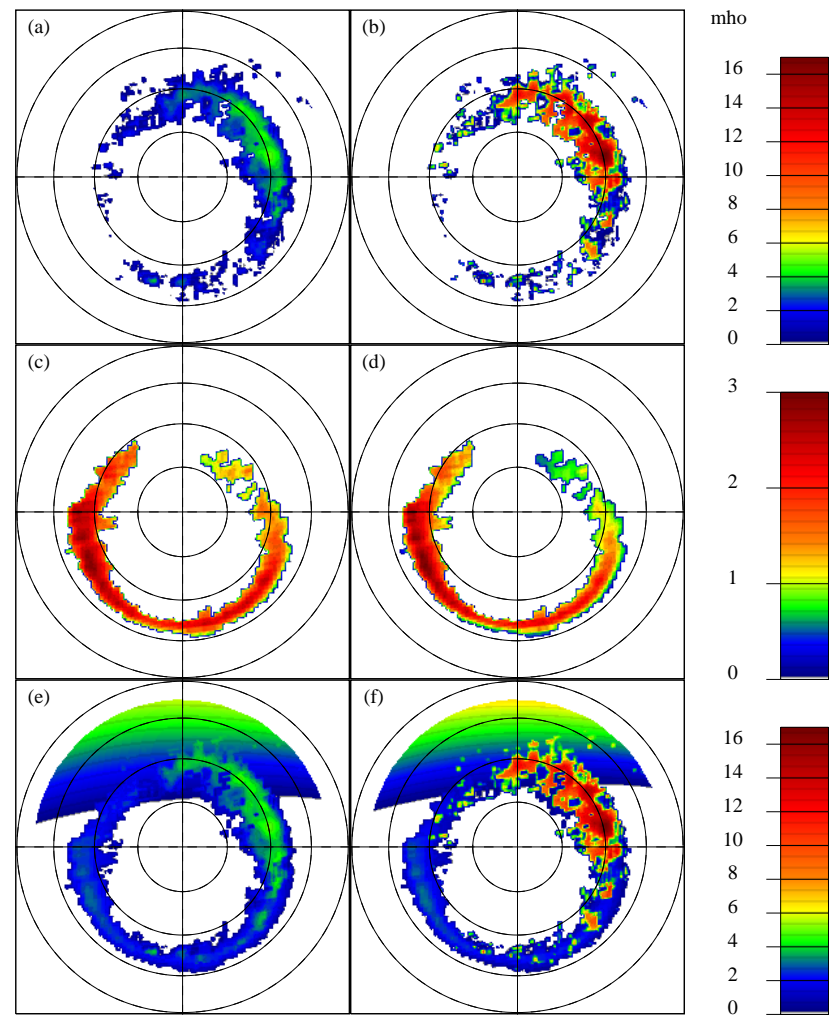

Plate 4. Conductance maps for 23 December 2000 21:04 UT (same as Plate 3) using electron mean energy computed from the ratio of WIC and SI13 simultaneous observations. Circles show $50^{\circ}, 60^{\circ}$, $70^{\circ}$ and $80^{\circ}$ MLAT. The local midnight is located downward.

Figure $4 c$ illustrates the influence of the use of FUV extracted electron mean energy on the calculated energy flux. Between 23:37:40 UT and 23:40:10 UT the energy flux from the mean energy of Hardy et al. (1987)'s model and from the WIC/SI13 ratio are very similar. Near 23:40:20 UT the energy flux shows a maximum up to $11 \mathrm{~mW} / \mathrm{m}^{2}$ which is induced by the peak in mean energy. The spatial resolution of the in situ data is much higher than the FUV instrumental resolution and therefore, in situ data were properly smoothed to account for this difference. Nevertheless, a difference between the theoretical and effective value may stem from the fact that in situ measurements are made along the orbital track, while FUV globally observes the auroral region. Each FUV pixel includes contributions from emissions adjacent to the low-latitude satellite track owing to its 2-3 pixel wide point spread function.

Figure $5 \mathrm{~b}$ showed the comparison between the electron mean energy calculated from FUV observations with the FAST measurements and the values from Hardy et al. (1985). Between 21:00:00 and 21:04:10 UT, the measured and the FUV evaluated mean energies follow the same evolution: they first increase until 21:02:10 UT and then decrease. However, the maximum energy in the measurements is about three times larger than the calculated one.

The WIC/SI13 ratio is a good global indicator of the morphology of the energy of auroral precipitation. The mean energy computed from the ratio is a good way to represent the spatial and the time evolution of the auroral electron precipitation. Fast and local fluctuations can be determined with the energy computed from this ratio while the Hardy et al. (1985)'s statistical mean energy cannot represent them. As discussed in Sect. 4.1.3, the uncertainties in the mean energy is the main source of error in the calculation of conductances.

Plates $4 \mathrm{a}$ and $\mathrm{b}$ show the electron induced conductance maps evaluated using the WIC/SI13 ratio to calculate the mean energy for the case of 23 December 2000. The peak in the Hall conductance reaches 17 mho and is thus globally higher than when calculated with the statistical mean energy (Plate 2b). The WIC observation shows two local maxima in the auroral precipitation in the sector between 00:00 and 06:00 MLT. They create two maxima in the Hall conductance not present in Plate $2 \mathrm{~b}$. The Pedersen conductance maps (Plates $4 \mathrm{a}$ and $2 \mathrm{a}$ ) induced by precipitating electrons show that the use of the mean energy computed from the FUV observations ( $4.9 \mathrm{mho}$ ) or statistical mean energy (5.4 mho) gives results quite similar. As discussed in Sect. 4.1.3, the error induced by the electron mean energy value is larger for the Hall than for the Pedersen conductance.

The upper limit set for the maximum mean energy is also a source of uncertainty. We evaluated the error on the conductance's when the electron mean energy is limited to $15 \mathrm{keV}$. If the mean energy was $20 \mathrm{keV}$, the error induced by the threshold would be $1.4 \%$ for the Hall and $\sim 18 \%$ for the Pedersen conductance. For increasing mean energy, the error increases for the Pedersen conductance and remains around $1.5 \%$ for the Hall conductance.

\section{Discussion and conclusions}

We developed a model using IMAGE-FUV observations to evaluate instantaneous ionospheric conductivity profiles and conductances induced by auroral precipitation on a global scale. The instantaneous Hall and Pedersen conductances produced by precipitating protons are computed on a global scale. The model requires assumptions on the auroral particles mean energy. We use the statistical models of Hardy et al. (1985) for the electron and Hardy et al. (1989) for the proton mean energies. During quiet periods the statistical model values are close to the actual particle mean energies. However, during substorms when the precipitation changes rapidly, the instantaneous variations in the auroral precipitation are not well represented by these statistical models. In these cases the ratio of simultaneous WIC and SI13 images may be used as an indicator of the electron energy. The morphology is then better represented but in some cases the quantitative values of the computed conductances are too high. The validity of using the precipitating particle characteristics from statistical model was tested by comparing the particle energy flux and mean energy evaluated from FUV observations with in situ measurements from the FAST and DMSP satellites. Comparisons show that the mean energies 
from Hardy et al. (1985; 1989)'s models are reasonably close to the in situ measurements. The electron mean energy deduced from the WIC to SI13 ratio often provides a better morphology than the statistical mean energy, meaning that the evolution of the mean energy and the energy flux along the low altitude satellite track are better reproduced. However the values are often overestimated, presumably as a consequence of the large sensitivity of the method to the background subtraction in the images.

As we discussed in Sect. 4, the main source of error in the conductance calculation is due to the uncertainties in the particle mean energy. Globally, the error in the Pedersen conductance is smaller than the one in the Hall conductance. Our calculated Pedersen conductance is thus more reliable than our calculated Hall conductance.

The application of the conductivity model to different cases for various magnetic activity level shows that the contribution of auroral protons in the total ionospheric conductances induced by auroral precipitation strongly depends on the location and mostly on the geomagnetic activity level. The contribution of protons is significant during quiet time periods. During substorms, the increase in conductances due to protons is globally less than $6 \%$, even though locally it can reach $\sim 10 \%$. During quiet time periods, the increase due to proton can reach globally up to $50 \%$ and locally nearly $100 \%$. In a study based on POLAR UVI images (Liou et al., 2001) protons were considered as electrons, as the UVI imager does not offer the possibility to discriminate between the two types of particles. We have calculated conductances without proton contribution in WIC and SI13 signals from two observations with different magnetic activity conditions. For the low $K_{p}$ case, the Pedersen and Hall conductances calculated, discriminating between electron and proton contribution $\left(\Sigma_{\mathrm{p}+\mathrm{e}}\right)$, can locally be 10 times higher than conductances calculated considering protons as electrons $\left(\Sigma_{\mathrm{p}=\mathrm{e}}\right)$, mainly in the dusk sector and for the equatorward limit of the auroral region, which reflects that the proton auroral oval is shifted equatorward of the electron oval in the dusk sector. For the case with higher $K_{p}$, the region where the difference is a factor 10 or more is much smaller. Over nearly all the auroral region, $\Sigma_{\mathrm{p}=\mathrm{e}}$ is nearly identical to $\Sigma_{\mathrm{p}+\mathrm{e}}$. This was expected during substorms as the electron precipitation is large.

The variations of auroral electron precipitation with season (Liou et al., 2001) or sunlight conditions (Newell et al., 1996) were interpreted in terms of an ionospheric conductivity feedback mechanism (Atkinson, 1970; Sato, 1978; Lysak, 1986). The feedback conductivity mechanism assumed that the ionospheric "background conductance" plays a role in the creation of the aurora. The ionosphere supports a large-scale electric field associated with plasma convection in the magnetosphere, which drives a large-scale electric current and the Pedersen part of which may be connected to a largescale field-aligned current. Precipitating particles create a density perturbation and an increase in the Pedersen conductivity. Depending on the ionosphere state, it reacts to this enhancement either as a voltage generator, or as a cur- rent generator. If the background ionospheric conductivity is high, the ionosphere responds by a polarization field which reduces the large-scale convection electric field. If the background conductivity is low, the ionosphere responds by an increase in the Pedersen current which must be closed by field-aligned currents flowing at the conductivity gradient. The field-aligned-current is associated with Alfvén waves (Lysak, 1986). More currents flow between the magnetosphere and the ionosphere. A field-aligned potential drop is required to pull more electrons from the low-density magnetosphere. It accelerates electrons and produces high energy electron precipitation and thus intense electron auroral arcs. As the electrons are accelerated by field-aligned electric field, protons are decelerated or accelerated upward. The observation of the seasonal variation of the upward ion beam was described in Collin et al. (1998). Observations with the TIMAS instrument on board the POLAR satellite are similar to the seasonal variations of the intense electron aurora and are in agreement with the mechanism. Moreover, observations from ground were also in the favor of the feedback conductivity mechanism. Nakano et al. (2002) analyzed local characteristics of the geomagnetic field to extract information on the field-aligned current variation. They showed that the net field-aligned currents are upward in the nightside region and downward in the dayside. In the nightside region, the background conductivity is low and the feedback mechanism implies that a more intense field-aligned current is flowing upward and the integration along the field line gives an important upward net field-aligned current.

We show that in some cases proton precipitation is very localized and very intense, as shown by the example of 28 April 2001. The increase in conductance due to protons is very high in this sector. The ionospheric conductivity feedback mechanism is plausibly also operative in this case. Proton precipitation produces a local increase in ionospheric conductivity which presumably causes more current to flow between the ionosphere and the magnetosphere and increases or decreases the field-aligned electric field and modifies the auroral precipitation. The ionosphere is primed for the feedback instability only when the background conductivity is low. In the case where proton precipitation is important, the background conductivity is high and the enhancement of Pedersen conductivity due to electron precipitation creates a polarization electric field, reducing the total convection electric field. The conditions are not suitable to create intense aurora. A very intense localized proton injection can influence the electron precipitation by reducing the electron acceleration. Thus, intense electron aurora is not expected to take place efficiently in regions where proton precipitation was important. The verification is beyond the scope of this study but the question requires further investigation. Lyons (1992) assumed that the potential variation which is the cause of the formation of electron auroral arcs generally occurs between about 5000 and $10000 \mathrm{~km}$ altitude. Can enhancement of ionospheric conductivity due to proton precipitation create an effect up to this altitude? 
Acknowledgements. V. Coumans is supported by a fellowship from the Belgian Fund for Research in Industry and Agriculture (FRIA) and J.-C. Gérard by the Belgian National Fund for Scientific Research (FNRS). This work was funded by the PRODEX program of the European Space Agency (ESA) and the Belgian Fund for Collective and Fundamental Research (FRFC grant \# 2.4517.02). We thank V. I. Shematovich and D. V. Bisikalo for their help in the determination of proton emission efficiencies. We acknowledge data provider, C. Carlson at U. C. Berkeley and CDAWeb for the FAST data.

Topical editor M. Lester thanks a referee for his help in evaluating this paper.

\section{References}

Atkinson, G.: Auroral arcs: Result of the interaction of a dynamic magnetosphere with the ionosphere, J. Geophys. Res., 75, 47464755, 1970.

Basu, B., Jasperse, J. R., Robinson, R. M., Vondrak, R. R., and Evans, D. S.: Linear transport theory of auroral proton precipitation: A comparison with observations, J. Geophys. Res., 92, 5920-5932, 1987.

Bilitza, D.: International Reference Ionosphere 1990, NSSDC 9022, Nat. Space Sci. Data Cent., Greenbelt, Md, 1990.

Brekke, A. and Hall, C.: Auroral ionospheric quiet summer time conductances, Ann. Geophys., 6, 361-376, 1988.

Brekke, A., Hall, C., and Hansen, T. L.: Auroral ionospheric conductances during disturbed conditions, Ann. Geophys., 7, 269280, 1989.

Burch, J. L.: Image Mission Overview, Space Science Reviews, 91, 1-14, 2000.

Codrescu, M. V., Fuller-Rowell, T. J., Roble, R. G., and Evans, D. S.: Medium energy particle precipitation influences on the mesosphere and lower thermosphere, J. Geophys. Res., 102, 1997719987, 1997.

Collin, H. L., Peterson, W. K., Lennartsson, O. W., and Drake, J. F.: The seasonal variation of the auroral ion beams, Geophys. Res. Lett., 25, 4071-4074, 1998.

Coumans, V., Gérard, J.-C., Hubert, B., and Evans, D. S.: Electron and proton excitation of the FUV aurora: Simultaneous IMAGE and NOAA observations, J. Geophys. Res., 107, 1347, doi:10.1029/2001JA009233, 2002.

Decker, D. T., Kozelov, B. V., Basu, B., Jasperse, J. R., and Ivanov, V. E.: Collisional degradation of the proton-H atom fluxes in the atmosphere: A comparison of theoretical techniques, J. Geophys. Res., 101, 26947-26960, 1996.

Evans, D. S., Maynard, N. C., Trøim, J., Jacobsen, T., and Egeland, A.: Auroral vector electric field and particle comparisons, 2. Electrodynamics of an arc, J. Geophys. Res., 82, 2235-2249, 1977.

Frey, H. U., Mende, S. B., Carlson, C. W., Gérard, J.-C., Hubert, B., Spann, J., Gladstone, R., and Immel, T. J.: The electron and proton aurora as seen by IMAGE-FUV and FAST, Geophys. Res. Lett., 28, 1135-1138, 2001.

Fuller-Rowell, T. J. and Evans, D. S.: Height-integrated Pedersen and Hall conductivity patterns inferred from the TIROS-NOAA satellite data, J. Geophys. Res., 92, 7606-7618, 1987.

Galand, M., Roble, R. G., and Lummerzheim, D.: Ionization by energetic protons in thermosphere-ionosphere electrodynamics general circulation model, J. Geophys. Res., 104, 27 973-27989, 1999.
Galand, M. and Richmond, A. D.: Ionospheric electrical conductances produced by auroral proton precipitation, J. Geophys. Res., 106, 117-126, 2001a.

Galand, M., Fuller-Rowell, T. J., and Codrescu, M. V.: Response of the upper atmosphere to auroral protons, J. Geophys. Res., 106, 127-139, 2001b.

Gérard, J.-C., Hubert, B., Bisikalo, D. V., and Shematovich, V. I.: A model of Lyman- $\alpha$ line profile in the proton aurora, J. Geophys. Res., 105, 15 795-15 805, 2000.

Gérard, J.-C., Hubert, B., Meurant, M., Shematovich, V. I., Bisikalo, D. V., Frey, H., Mende, S., Gladstone, G. R., and Carlson, C. W.: Observation of the proton aurora with IMAGE FUV imager and simultaneous ion flux in situ measurements, J. Geophys. Res., 106, 28 939-28 948, 2001.

Gjerloev, J. W. and Hoffman, R. A.: Height-integrated conductivity in auroral substorms, 1. Data, J. Geophys. Res., 105, 215-226, 2000a.

Gjerloev, J. W. and Hoffman, R. A.: Erratum: "Height-integrated conductivity in auroral substorms, 1. Data", J. Geophys. Res., 105, 10675-10 676, 2000b.

Gjerloev, J. W. and Hoffman, R. A.: Height-integrated conductivity in auroral substorms, 2. Modeling, J. Geophys. Res., 105, 227235, 2000c.

Hardy, D. A., Gussenhoven, M. S., and Holeman, E.: A statistical model of auroral electron precipitation, J. Geophys. Res., 90, 4229-4248, 1985.

Hardy, D. A., Gussenhoven, M. S., Raistrick, R., and McNeil, W. J.: Statistical and functional representations of the pattern of auroral energy flux, number flux, and conductivity, J. Geophys. Res., 92, 12 275-12 294, 1987.

Hardy, D. A., Gussenhoven, M. S., and Brautigam, D.: A statistical model of auroral ion precipitation, J. Geophys. Res., 94, 370392, 1989.

Hardy, D. A., McNeil, W., Gussenhoven, M. S., and Brautigam, D.: A statistical model of auroral ion precipitation, 2. Functional representation of the average patterns, J. Geophys. Res., 96, 55395548, 1991.

Hedin, A. E.: Extension of the MSIS thermosphere model into the middle and lower atmosphere, J. Geophys. Res., 96, 1159-1172, 1991.

Horwitz, J. L., Doupnik, J. R., and Banks, P. M.: Chatanika radar observations of the latitudinal distributions of auroral zone electric fields, conductivities, and currents, J. Geophys. Res., 83, 1463-1481, 1978.

Hubert, B., Gérard, J. C., Bisikalo, D. V., Shematovich, V. I., and Solomon, S. C.: The role of proton precipitation in the excitation of the auroral FUV emissions, J. Geophys. Res., 106 , 21 47521 494, 2001.

Hubert, B., Gérard, J. C., Evans, D. S., Meurant, M., Mende, S. B., Frey, H. U., and Immel, T. J.: Total electron and proton energy input during auroral substorms: Remote sensing with IMAGEFUV, J. Geophys. Res., 107, doi:10.1029/2001JA009229, 2002.

Immel, T., Craven, J. D., and Nicholas, A. C.: An empirical model of the OI FUV dayglow from DE-1 images, J. Atmos. Sol.-Terr. Phy., 62, 47-64, 2000.

Inhester, B., Untiedt, J., Segatz, M., and Kürschner, M.: Direct determination of the local ionospheric hall conductance distribution from two-dimensional electric and magnetic field data, J. Geophys. Res., 97, 4073-4084, 1992.

Kosch, M. J., Hagfors, T., and Schlegel, K.: Extrapolating EISCAT Pedersen conductances to other parts of the sky using groundbased TV auroral images, Ann. Geophys., 16, 583-588, 1998. 
Lester, M., Davies, J. A., and Virdi, S.: High-latitude Hall and Pedersen conductances during substorm activity in the SUNDIALATLAS campaign, J. Geophys. Res., 101, 2671926 728, 1996.

Liou, K., Newell, P. T., and Meng, C.-I: Seasonal effects on auroral particle acceleration and precipitation, J. Geophys. Res., 106, 5531-5542, 2001.

Lummerzheim, D., Rees, M. H., Craven, D. J., and Frank L. A.: Ionospheric conductances derived from DE-1 auroral images, J. Atm. Terr. Phys., 53, 281-292, 1991.

Lyons, L. R.: Formation of auroral arcs via magnetosphereionosphere coupling, Rev. Geophys., 30, 93-112, 1992.

Lysak, R. L.: Coupling of the dynamic ionosphere to auroral flux tubes, J. Geophys. Res., 91, 7047-7056, 1986.

Marov, M. Y., Shematovich, V. I., Bisikalo, D. V., and Gérard, J.C.: Nonequilibrium processes in planetary and cometary atmosphere: Theory and applications, Kluwer Acad., Norwell, Mass., 1997.

Mende, S. B., Vondrak, R. R., Eather, R. H., Rees, M. H., and Robinson, R. M.: Optical mapping of ionospheric conductance, J. Geophys. Res., 89, 1757-1767, 1984.

Mende, S. B., Heetderks, H., Frey, H. U., Lampton, M., Geller, S. P., Habraken, S., Renotte, E., Jamar, C., Rochus, P., Spann, J., Fuselier, S. A., Gérard, J.-C., Gladstone, R., Murphree, S., and Cogger, L.: Far ultraviolet imaging from the IMAGE spacecraft, 1. System Design, Space Science Reviews, 91, 243-270, 2000.

Meurant, M., Gérard, J.-C., Hubert, B., Coumans, V., Shematovich, V. I., Bisikalo, D. V., Gladstone, R., Evans, D. S., and Mende, S. B.: Characterization of the auroral electron precipitation energy during substorms from the IMAGE-FUV imagers and application, J. Geophys. Res., 2003.

Nakano, S., Iyemori, T., and Yamashita, S.,: Net field-aligned currents controlled by the polar ionospheric conductivity, J. Geophys. Res., 107, doi:10.1029/2001JA900177, 2002.

Newell, P. T., Meng, C.-I., and Lyons, K. M.: Suppression of discrete aurorae by sunlight, Nature, 381, 766-767, 1996.

Olsen, N., Sabaka, T. J., and Tøffner-Clausen, L.: Determination of the IGRF 2000 model, Earth Planets Space, 52, 1175-1182, 2000.

Rasmussen, C. E., Schunk, R. W., and Wickwar, V. B.: A photochemical equilibrium model for ionospheric conductivity, J. Geophys. Res., 93, 9831-9840, 1988.
Rees, M. H., Lummerzheim, D., Roble, R. G., Winningham, J. D., Craven, J. D. and Frank, L. A.: Auroral energy deposition rate, characteristic electron energy, and ionospheric parameters derived from dynamics explorer, 1. Images, J. Geophys. Res., 93 12 841-12860, 1988.

Rees, M. H.: Physics and chemistry of the upper atmosphere, Cambridge Univ. Press, New York, 1989.

Rishbeth, H. and Garriott, O. K.: Introduction to ionospheric physics, Academic Press, New York and London, 1969.

Robinson, R. M., Vondrak, R. R., Miller, K., Dabbs, T., and Hardy, D.: On calculating ionospheric conductances from the flux and energy of precipitating electrons, J. Geophys. Res., 92, 25652569, 1987.

Robinson, R. M., Vondrak, R. R., Craven, J. D., Frank, L. A., and Miller, K.: A comparison of ionospheric conductances and auroral luminosities observed simultaneously with the Chatanika radar and the DE 1 auroral imagers, J. Geophys. Res., 94, 53825396, 1989.

Sato, T.: A theory of quiet auroral arcs, J. Geophys. Res., 83, 10421047, 1978.

Schlegel, K.: Auroral zone E-region conductivities during solar minimum derived from EISCAT data, Ann. Geophys., 6, 129138, 1988.

Solomon, S. C., Hays, P. B., and Abreu, V.: The auroral 6300 Å emission: observation and modeling, J. Geophys. Res., 93, 9867-9882, 1988

Spiro, R. W., Reiff, P. H., and Maher, L. J.: Precipitating electron energy flux and auroral zone conductances - an empirical model, J. Geophys. Res., 87, 8215, 1982.

Vickrey, J. F., Vondrak, R. R., and Matthews, S. J.: The diurnal and latitudinal variation of auroral zone ionospheric conductivity, J. Geophys. Res., 86, 65-75, 1981.

Vickrey, J. F., Vondrak, R. R., and Matthews, S. J.: Energy deposition by precipitating particles and joule dissipation in the auroral ionosphere, J. Geophys. Res., 87, 5184-5196, 1982.

Vondrak, R. R. and Robinson, R. M.: Inference of high-latitude ionization and conductivity from AE-C measurements of auroral electron fluxes, J. Geophys. Res., 90, 7505-7512, 1985.

Wallis, D. D. and Budzinski, E. E.: Empirical models of height integrated conductivities, J. Geophys. Res., 86, 125-138, 1981. 